\title{
Hilbert Space Sectors for Solutions of Non-linear Relativistic Field Equations
}

\author{
C. Parenti` \\ Istituto Matematico "S. Pincherle", University of Bologna, Bologna, Italy \\ F. Strocchi \\ Scuola Normale Superiore and I.N.F.N., Sez. di Pisa, Pisa, Italy \\ G. Velo \\ Istituto di Fisica “A. Righi”, University of Bologna and I.N.F.N., Sez. di Bologna, Bologna, Italy
}

\begin{abstract}
In the set of Cauchy data corresponding to the solutions of nonlinear classical relativistic field equations having locally finite kinetic energy a structure of Hilbert space sectors is introduced. Each sector is invariant under time evolution and a total energy and linear momentum functionals can be defined as global quantities. Within this framework the existence of conserved dynamical charges is proved and the mechanism by which a symmetry can be spontaneously broken is ex plained.
\end{abstract}

\section{Introduction}

Recently there has been a revived interest in solutions of classical non-linear field equations as a means of understanding basic properties of elementary particles [1]. The main idea is to classify some simple stable solutions of field equations and to analyze small perturbations around them. Examples of such solutions are the constants which minimize the energy [2] and the solitons [3]. One hopes that essential features coming from the non-linear character of the theory are taken care of by the structure of those special solutions. This approach to non-linear field theory is also crucial for understanding spontaneous symmetry breaking [2], stability problems and for explaining the occurrence of charges which are of dynamical rather than of group-theoretical origin [4]. Moreover the properties of classical solutions of non-linear field equations are relevant for the quantum field theory version since they correspond to the expectation values of quantum fields on suitable coherent states [5]. These ideas look interesting and promising and deserve a systematic investigation, which seems to be lacking in the literature.

The purpose of this paper is to provide a rigorous treatment of some non-linear systems of partial differential equations for classical fields along the above lines. We shall be able to answer a certain number of questions like the construction

* Partially supported by C.N.R. (gruppo G.N.A.F.A.) 
of Hilbert space sectors, their stability under time evolution, the existence of dynamical charges and the occurrence of spontaneous symmetry breaking.

More specifically we will study the solutions of the following Cauchy problem

$$
\begin{aligned}
& \square \varphi_{k}(x, t)+\frac{\partial U}{\partial \varphi_{k}}\left(x, \varphi_{1}(x, t), \ldots, \varphi_{n}(x, t)\right)=0, \\
& \forall(x, t) \in \mathbb{R}^{s} \times \mathbb{R}, \quad k=1, \ldots, n \\
& \varphi_{k}(x, 0)=\varphi_{0 k}(x) \\
& \frac{\partial \varphi_{k}}{\partial t}(x, 0)=\psi_{0 k}(x)
\end{aligned}
$$

which can more conventiently be rewritten as the integral equation

$$
\left(\begin{array}{c}
\varphi(t) \\
\psi(t)
\end{array}\right)=W(t)\left(\begin{array}{c}
\varphi_{0} \\
\psi_{0}
\end{array}\right)+\int_{0}^{t} W(t-s)\left(\begin{array}{c}
0 \\
-\nabla U(x, \varphi(s))
\end{array}\right) d s
$$

where $\left(\begin{array}{c}\varphi \\ \psi\end{array}\right)=\left(\begin{array}{c}\varphi_{1} \\ \psi_{1} \\ \vdots \\ \varphi_{n} \\ \psi_{n}\end{array}\right)$ and $W(t)$ is the one parameter group generated by $\left(\begin{array}{ll}0 & 1 \\ \Delta & 0\end{array}\right) \otimes \mathbb{1}_{\mathbb{C}^{n}}$. In a preceding paper [6] we have proved existence, uniqueness and regularity theorems for Equation (0.2), under suitable conditions on the potential $U$ (see Section 1), in the space $X$ of real functions having locally finite kinetic energy. We have shown that if $\left(\begin{array}{l}\varphi_{0} \\ \psi_{0}\end{array}\right) \in X$, the integral Equation (0.2) has a unique $X$-valued continuous solution $\left(\begin{array}{l}\varphi(t) \\ \psi(t)\end{array}\right) \in C^{(0)}(\mathbb{R}, X)$, where

$$
X=X_{1} \oplus X_{0}, \quad X_{0}=\bigoplus_{n} L_{\mathrm{loc}}^{2}\left(\mathbb{R}^{s}\right), \quad X_{1}=\bigoplus_{n} H_{\mathrm{loc}}^{1}\left(\mathbb{R}^{s}\right)
$$

Here we propose to continue the analysis started in [6] by investigating some finer structure properties of the family $\mathscr{F}$ of all solutions $\left(\begin{array}{l}\varphi(t) \\ \psi(t)\end{array}\right) \in C^{(0)}(\mathbb{R}, X)$ of Equation (0.2). In order to have a simple physical interpretation of the theory it is convenient to partition the set $\mathscr{F}$ into classes in such a way that solutions belonging to the same class have relatively finite energy. It is clear, in fact, that initial data, for which the energy difference of the corresponding solutions is infinite, cannot be realized in the same "physical world". It is therefore reasonable to introduce an equivalence relation between the elements of $\mathscr{F}$ in the following way

$$
\left(\begin{array}{l}
\varphi(t) \\
\psi(t)
\end{array}\right) \sim\left(\begin{array}{c}
\varphi^{\prime}(t) \\
\psi^{\prime}(t)
\end{array}\right) \quad \text { iff } \quad\left(\begin{array}{c}
\varphi(t)-\varphi^{\prime}(t) \\
\psi(t)-\psi^{\prime}(t)
\end{array}\right) \in C^{(0)}(\mathbb{R}, Y)
$$


where

$$
Y=Y_{1} \oplus Y_{0}, \quad Y_{0}=\bigoplus_{n} L^{2}\left(\mathbb{R}^{s}\right), \quad Y_{1}=\bigoplus_{n} H^{1}\left(\mathbb{R}^{s}\right)
$$

As a further physical requirement we consider those classes for which each of its elements $\left(\begin{array}{l}\varphi(t) \\ \psi(t)\end{array}\right)$ has the property that

$$
\left(\begin{array}{c}
\varphi(t)-\varphi(0) \\
\psi(t)-\psi(0)
\end{array}\right) \in C^{(0)}(\mathbb{R}, Y)
$$

This amounts to restricting ourselves to the classes which are left invariant by the time translations. Relation (0.4) together with condition (0.6) allows us to introduce Hilbert space sectors in the set $X$ of initial data. Two elements of $X$ belong to the same Hilbert sector iff the corresponding solutions are equivalent in the sense of relation (0.4) and satisfy condition (0.6). Obviously, each Hilbert sector is uniquely determined by any of its elements $\left(\begin{array}{c}\varphi \\ \psi\end{array}\right)$, and will be denoted by $\mathscr{H}_{(\varphi, \psi)}$.

The above structure finds a natural justification in the following theorems (which will be proved in Section 2 under suitable conditions on the potential $U$ ).

Theorem A. If a solution $\left(\begin{array}{l}\varphi(t) \\ \psi(t)\end{array}\right)$ of Equation (0.2) satisfies condition (0.6) and $\varphi_{0 k} \in L^{\infty}\left(\mathbb{R}^{s}\right), k=1, \ldots, n$, then

$$
\begin{aligned}
& \Delta \varphi_{0 k}-\frac{\partial U}{\partial \varphi_{k}}\left(x, \varphi_{01}, \ldots, \varphi_{0 n}\right) \in H^{-1}\left(\mathbb{R}^{s}\right), \\
& \psi_{0 k} \in L^{2}\left(\mathbb{R}^{s}\right), \quad k=1, \ldots, n .
\end{aligned}
$$

Theorem B. If $\left(\begin{array}{c}\varphi \\ \psi\end{array}\right) \in X$ satisfies the following conditions
i) $\Delta \varphi_{k}-\frac{\partial U}{\partial \varphi_{k}}\left(x, \varphi_{1}, \ldots, \varphi_{n}\right) \in H^{-1}\left(\mathbb{R}^{s}\right)$,
$\left.\begin{array}{rl}\text { ii) } \varphi_{k} \in L^{\infty}\left(\mathbb{R}^{s}\right) \\ \text { iii) } \psi_{k} \in L^{2}\left(\mathbb{R}^{s}\right)\end{array}\right\} k=1, \ldots, n$,

then $\left(\begin{array}{l}\varphi \\ \psi\end{array}\right)$ determines a Hilbert space sector, whose elements are all the $\left(\begin{array}{l}\varphi^{\prime} \\ \psi^{\prime}\end{array}\right) \in X$ such that $\left(\begin{array}{c}\varphi-\varphi^{\prime} \\ \psi-\psi^{\prime}\end{array}\right) \in Y$.

Condition i) of Theorem B is obviously fulfilled by the static solutions of Equation (0.1). In particular, the elements of $X$ of the form $\left(\begin{array}{c}\varphi=\text { const } \\ \psi=0\end{array}\right)$ determine Hilbert sectors iff $\frac{\partial U}{\partial \varphi_{k}}(x$, const $)=0, k=1, \ldots, n$, i.e. if they extremize the potential (Goldstone's theorem [2]). 
Furthermore, for each Hilbert sector $\mathscr{H}_{(\varphi, \varphi)}$ such that $\varphi_{k} \in L^{\infty}\left(\mathbb{R}^{s}\right), \nabla \varphi_{k} \in L^{2}\left(\mathbb{R}^{s}\right)$, $k=1, \ldots, n$, one may define a total energy functional

$$
\mathscr{H}_{(\varphi, \psi)} \ni\left(\begin{array}{c}
\varphi^{\prime} \\
\psi^{\prime}
\end{array}\right) \rightarrow E_{(\varphi, \psi)}\left(\varphi^{\prime}, \psi^{\prime}\right)=\lim _{R \rightarrow+\infty} \int_{\mathbb{R}^{s}}\left[K\left(\varphi^{\prime}, \psi^{\prime}\right)-K(\varphi, \psi)\right] \omega\left(\frac{x-x_{0}}{R}\right) d x
$$

where

$$
K(\alpha, \beta)=\sum_{j=1}^{n} \frac{\left|\nabla \alpha_{j}\right|^{2}+\beta_{j}^{2}}{2}+U(x, \alpha),
$$

$\omega$ is a test function, belonging to $C_{0}^{\infty}\left(\mathbb{R}^{s}\right)$, equal to 1 in a neighbourhood of the origin, and $x_{0} \in \mathbb{R}^{s}$. The functional (0.7) is independent of $\omega$ and of $x_{0}$, invariant under time translations and satisfies the identity

$$
E_{(\varphi, \psi)}\left(\varphi^{\prime}, \psi^{\prime}\right)=E_{(\alpha, \beta)}\left(\varphi^{\prime}, \psi^{\prime}\right)+E_{(\varphi, \psi)}(\alpha, \beta)
$$

if $\left(\begin{array}{c}\alpha \\ \beta\end{array}\right) \in \mathscr{H}_{(\varphi, \psi)}$ and $\alpha_{k} \in L^{\infty}\left(\mathbb{R}^{s}\right), k=1, \ldots, n$ (see Section 3).

Identity (0.9) shows that within such sectors the energy scale does not have a physical meaning. In the same Hilbert sectors one may define a linear momentum functional

$$
\boldsymbol{P}_{(\varphi, \psi)}\left(\varphi^{\prime}, \psi^{\prime}\right)=\int_{\mathbb{R}^{s}} \sum_{j=1}^{n}\left[\psi_{j}^{\prime} \nabla \varphi_{j}^{\prime}-\psi_{j} \nabla \varphi_{j}\right] d x
$$

which is obviously finite.

Within this framework we can prove the existence of dynamical charges which are constant in time. The key observation is that if $\varphi \in Y_{1}$, then $\tilde{\varphi}(r, \omega) \equiv$ $\varphi(x=r \omega) \rightarrow 0$ as $r \rightarrow+\infty$ for almost all $\omega \in \mathbb{S}^{s-1}$. This shows that all the elements of a Hilbert sector have the same asymptotic behaviour as $r \rightarrow+\infty$ (almost everywhere in $\mathbb{S}^{s-1}$ ) and that this behaviour is preserved in time. Furthermore, for sectors $\mathscr{H}_{(\varphi, \psi)}$ with $\nabla \varphi_{k} \in L^{2}\left(\mathbb{R}^{s}\right), k=1, \ldots, n$ and for $s \geqq 3$, we may prove that $\tilde{\varphi}(r, \omega)$ has a finite limit $a(\omega)$, as $r \rightarrow+\infty$, for almost all $\omega \in \mathbb{S}^{s-1}$. Analogous results hold for $s=1$ under suitable conditions on the potentials $U$ (see Appendix C). Each function $a(\omega)$ identifies a charge whose origin is strictly related to the structure of the Hilbert sectors, i.e. to the dynamics of the theory (dynamical charges).

In the space $X$ one may introduce the concept of local internal symmetry and the existence of Hilbert sectors explains the mechanism by which a local internal symmetry can be spontaneously broken in a given sector. Local internal symmetries $g$ are proved to be affine transformations (see Section 5) $g(\varphi)=A_{g} \varphi+a$, with $A_{g}^{T} A_{g}=\lambda_{g} \mathbb{1}_{\mathbb{R}^{n}}$, and can be described by unitary operators in a given sector if they are not spontaneously broken in that sector.

The plan of the paper is as follows:

0 . Introduction

1. General framework

2. Hilbert space sectors-Stability and time evolution

3. Total energy and momentum

4. Dynamical charges

5. Internal symmetries and spontaneous symmetry breaking

Appendix A, B, C 
Finally we list the non-standard symbols used to denote some functional spaces.

$$
\begin{aligned}
Y_{0} & =\bigoplus_{n} L^{2}\left(\mathbb{R}^{s}\right), \quad\left\|\zeta ; Y_{0}\right\|=\left(\sum_{j=1}^{n}\left\|\zeta_{j} ; L^{2}\left(\mathbb{R}^{s}\right)\right\|^{2}\right)^{1 / 2}, \\
Y_{1} & =\bigoplus_{n} H^{1}\left(\mathbb{R}^{s}\right), \quad\left\|\chi ; Y_{1}\right\|=\left(\sum_{j=1}^{n}\left\|\chi_{j} ; H^{1}\left(\mathbb{R}^{s}\right)\right\|^{2}\right)^{1 / 2}, \\
Y & =Y_{1} \oplus Y_{0} . \\
X_{0} & =\bigoplus_{n} L_{\mathrm{loc}}^{2}\left(\mathbb{R}^{s}\right), \quad\left\|\psi ; X_{0}(\Omega)\right\|=\left(\sum_{j=1}^{n}\left\|\psi_{j} ; L^{2}(\Omega)\right\|^{2}\right)^{1 / 2}, \\
X_{1} & =\bigoplus_{n} H_{\mathrm{loc}}^{1}\left(\mathbb{R}^{s}\right), \quad\left\|\varphi ; X_{1}(\Omega)\right\|=\left(\sum_{j=1}^{n}\left\|\varphi_{j} ; H^{1}(\Omega)\right\|^{2}\right)^{1 / 2}, \\
X & =X_{1} \oplus X_{0} .
\end{aligned}
$$

( $\Omega$ is any open bounded set).

\section{General Framework}

We state existence and uniqueness theorems, for the integral Equation (0.2), which will be needed in the following.

Theorem 1. Let be given a function $U(x, z): \mathbb{R}^{s} \times \mathbb{R}^{n} \rightarrow \mathbb{R}$ of class $C^{(2)}$ in the $z$ variable satisfying the conditions

1) $\nabla_{z} U(x, \varphi) \in X_{0}, \forall \varphi \in X_{1}$.

2) For any sphere $\Omega_{R}{ }^{1}$ of radius $R$ and for any $\varrho>0$ there exists a positive constant $C\left(\Omega_{R}, \varrho\right)$, for which $\sup _{0 \leqq t \leqq R / 2} C\left(\Omega_{R-t}, \varrho\right)<\infty$, and such that

$$
\left\|\nabla_{z} U\left(x, \varphi^{(1)}\right)-\nabla_{z} U\left(x, \varphi^{(2)}\right) ; X_{0}\left(\Omega_{R}\right)\right\| \leqq C\left(\Omega_{R}, \varrho\right)\left\|\varphi^{(1)}-\varphi^{(2)} ; X_{1}\left(\Omega_{R}\right)\right\|,
$$

for all $\varphi^{(k)} \in X_{1},\left\|\varphi^{(k)} ; X_{1}\left(\Omega_{R}\right)\right\| \leqq \varrho, k=1,2$.

Then, for any initial data $\left(\begin{array}{l}\varphi \\ \psi\end{array}\right) \in X$ the integral equation

$$
\left(\begin{array}{l}
\varphi(t) \\
\psi(t)
\end{array}\right)=W(t)\left(\begin{array}{l}
\varphi \\
\psi
\end{array}\right)+\int_{0}^{t} W(t-s)\left(\begin{array}{c}
0 \\
\left.-\nabla_{z} U(x, \varphi(s))\right)
\end{array}\right) d s
$$

where $W(t)$ is the one parameter group in $X$ generated by $\left(\begin{array}{ll}0 & 1 \\ \Delta & 0\end{array}\right) \otimes \mathbb{1}_{\mathbb{C}^{n}}$, has at most one solution $\left(\begin{array}{l}\varphi(t) \\ \psi(t)\end{array}\right) \in C^{0)}(\mathbb{R}, X)$.

Theorem 2. Under the same conditions on $U$ stated in Theorem 1 and the additional assumption

3) There exist two non negative continuous functions $\alpha, \beta: \mathbb{R}^{s} \rightarrow \mathbb{R}$ such that

$$
U(x, z) \geqq-\alpha(x)-\beta(x)|z|^{2}, \quad(x, z) \in \mathbb{R}^{s} \times \mathbb{R}^{n},
$$

the integral Equation (1.2) has a solution $\left(\begin{array}{l}\varphi(t) \\ \psi(t)\end{array}\right) \in C^{(0)}(\mathbb{R}, X)$ for any initial data in $X$.

1 In this paper sphere will be synonymous of open sphere; $\Omega_{R-t}$ will denote the sphere of radius $R-t$ concentric to $\Omega_{R}$ 
Theorems 1 and 2 are essentially contained in Theorem 3.2 of [6], with some minor modifications we are going to ex plain. In contrast to what was done in [6], where $\nabla_{z} U(x, z)$ was split into three terms called $j, g^{(1)}$ and $g^{(2)}$ and suitable conditions were imposed separately on each term, here the conditions are directly given on $U$ and $\nabla_{z} U$. To perform a kind of identification we could take $g^{(2)}(x, z)=$ $2 \beta(x) z, j(x, z)+g^{(1)}(x, z)=-\nabla_{z} U(x, z)-2 \beta(x) z$, and $G(x, z)=U(x, z)+\beta(x)|z|^{2}$. The only difference with respect to Definitions 3.1 and 3.4 of [6] is that now $G(x, z)$ and $\nabla_{z} G(x, z)$ do not necessarily vanish for $z=0$. Furthermore, assumption iii) of Definition 3.1 is replaced here by the slightly weaker assumption 2 ) of Theorem 1 . The proof of Theorems 1 and 2 will not be given here since it involves only trivial changes in the proof of Theorem 3.2 of [6].

In the next sections we will analyze finer structure properties of the family $\mathscr{F}$ of solutions of Equation (1.2) belonging to the space $C^{0)}(\mathbb{R}, X)$. In particular we will state necessary and sufficient conditions for the ex istence of Hilbert space sectors. For this purpose one is naturally lead to study the integral equation satisfied by the vector valued function $\left(\begin{array}{l}\chi(t) \\ \zeta(t)\end{array}\right), \chi(t)=\varphi^{\prime}(t)-\varphi, \zeta(t)=\psi^{\prime}(t)-\psi$, where $\left(\begin{array}{l}\varphi^{\prime}(t) \\ \psi^{\prime}(t)\end{array}\right)$ belongs to $\mathscr{F}$ and $\varphi^{\prime}(0)-\varphi \in Y_{1}, \psi^{\prime}(0)-\psi \in Y_{0}$. Such integral equation has the following form (for a derivation see Section 2):

$$
\begin{aligned}
\left(\begin{array}{l}
\chi(t) \\
\zeta(t)
\end{array}\right)= & W(t)\left(\begin{array}{l}
\varphi^{\prime}(0)-\varphi \\
\psi^{\prime}(0)-\psi
\end{array}\right)+(W(t)-\mathbb{1})\left(\begin{array}{l}
\varphi \\
\psi
\end{array}\right) \\
& +\int_{0}^{t} W(t-s)\left(\begin{array}{c}
0 \\
-\nabla_{z} U(x, \varphi)
\end{array}\right) d s+\int_{0}^{t} W(t-s)\left(\begin{array}{c}
0 \\
-\nabla_{z} F_{\varphi}(x, \chi(s))
\end{array}\right) d s
\end{aligned}
$$

where

$$
F_{\varphi}(x, z)=U(x, \varphi(x)+z)-U(x, \varphi(x))-\sum_{j=1}^{n} \frac{\partial U}{\partial z_{j}}(x, \varphi(x)) z_{j} .
$$

The crucial step is to prove that Equation (1.4) has a unique solution

$$
\left(\begin{array}{l}
\chi(t) \\
\zeta(t)
\end{array}\right) \in C^{(0)}(\mathbb{R}, Y)
$$

This will be done by a slight modification of Segal's approach [7], which involves Lipschitz and "positivity" conditions on $F_{\varphi}$. In this section we will establish some preliminary results connecting Lipschitz and positivity conditions of $F_{\varphi}$ to corresponding properties of $U$.

Definition 1. We will say that a map

$$
f: \mathbb{R}^{s} \times X_{1} \rightarrow \mathscr{D}^{\prime}\left(\mathbb{R}^{s}\right)
$$

is locally Lipschitz continuous if for any sphere $\Omega_{R}$ of radius $R$ and for any $\varrho>0$ there exists a positive constant $C\left(\Omega_{R}, \varrho\right)$, for which $\sup _{0 \leqq t \leqq R / 2} C\left(\Omega_{R-t}, \varrho\right)<\infty$, such that

$$
\left\|f\left(x, \varphi^{(1)}\right)-f\left(x, \varphi^{(2)}\right) ; X_{0}\left(\Omega_{R}\right)\right\| \leqq C\left(\Omega_{R}, \varrho\right)\left\|\varphi^{(1)}-\varphi^{(2)} ; X_{1}\left(\Omega_{R}\right)\right\|
$$


for all

$$
\varphi^{(k)} \in X_{1}, \quad\left\|\varphi^{(k)} ; X_{1}\left(\Omega_{R}\right)\right\| \leqq \varrho, \quad k=1,2 .
$$

We will say that a map

$$
f: \mathbb{R}^{s} \times Y_{1} \rightarrow \mathscr{D}^{\prime}\left(\mathbb{R}^{s}\right)
$$

is globally Lipschitz continuous if for any $\varrho>0$ there exists a positive constant $C(\varrho)$ such that

$$
\left\|f\left(x, \chi^{(1)}\right)-f\left(x, \chi^{(2)}\right) ; Y_{0}\right\| \leqq C(\varrho)\left\|\chi^{(1)}-\chi^{(2)} ; Y_{1}\right\|
$$

for all

$$
\chi^{(k)} \in Y_{1},\left\|\chi^{(k)} ; Y_{1}\right\| \leqq \varrho, \quad k=1,2 .
$$

Definition 2. By $\mathscr{U}(X)$ we denote the class of all maps $U(x, z): \mathbb{R}^{s} \times \mathbb{R}^{n} \rightarrow \mathbb{R}$ of class $C^{(2)}$ in the $z$-variables with the properties

i) $\nabla_{z} U(x, \varphi) \in X_{0}, \forall \varphi \in X_{1}$.

ii) The map $\mathbb{R}^{s} \times X_{1} \ni(x, \varphi) \mapsto \nabla_{z} U(x, \varphi)$ is locally Lipschitz continuous.

iii) There exist two non-negative constants $\alpha, \beta$ such that

$$
U(x, z) \geqq-\alpha-\beta|z|^{2}, \quad(x, z) \in \mathbb{R}^{s} \times \mathbb{R}^{n} .
$$

By $\mathscr{U}(Y)$ we denote the class of all maps $F(x, z): \mathbb{R}^{s} \times \mathbb{R}^{n} \rightarrow \mathbb{R}$ of class $C^{(2)}$ in the $z$-variables with the properties

i) $\nabla_{z} F(x, \chi) \in Y_{0}, \forall \chi \in Y_{1}$.

ii) ${ }^{\prime}$ The map $\mathbb{R}^{s} \times Y_{1} \ni(x, \chi) \mapsto \nabla_{z} F(x, \chi)$ is globally Lipschitz continuous.

iii) $)^{\prime}$ There exists a non negative constant $\gamma$ such that

$$
F(x, z) \geqq-\gamma|z|^{2}, \quad(x, z) \in \mathbb{R}^{s} \times \mathbb{R}^{n} .
$$

Clearly each $U \in \mathscr{U}(X)$ satisfies the hypotheses of Theorems 1 and 2. A reason for defining the class $\mathscr{U}(Y)$ is that if $F_{\varphi}$ [see Eq. (1.5)] belongs to $\mathscr{U}(Y)$ then, under suitable assumptions on $\varphi$ and $\psi$, Equation (1.4) has a unique solution in $Y$ (see Section 2). We remark that $\mathscr{U}(Y) \subset \mathscr{U}(X)$ as a consequence of the following

Lemma 1. Let be given a function $g(x, z): \mathbb{R}^{s} \times \mathbb{R}^{n} \rightarrow \mathbb{R}^{n}$.

Then

1) If $g(x, \chi) \in Y_{0} \forall \chi \in Y_{1}$, it follows that $g(x, \varphi) \in X_{0} \forall \varphi \in X_{1}$;

2) If the map $\mathbb{R}^{s} \times Y_{1} \ni(x, \chi) \mapsto g(x, \chi)$ is globally Lipschitz continuous it is also locally Lipschitz continuous.

Proof. For a given sphere $\Omega_{R}$ let $\pi_{R}: \bigoplus_{n} H^{1}\left(\Omega_{R}\right) \rightarrow Y_{1}$ denote a linear continuous extension operator with the following property (see e.g. Appendix B of [6]): There is a function $d(R)$ which is $O(R)$ if $R \geqq 1$ and $0\left(R^{-1}\right)$ if $R<1$, such that $\left\|\pi_{R}\right\| \leqq d(R)$.

Suppose now that $g(x, \chi) \in Y_{0}$ if $\chi \in Y_{1}$. The locality of $g$ yields

$$
\begin{aligned}
\left\|g(x, \varphi) ; X_{0}\left(\Omega_{R}\right)\right\| & =\left\|g\left(x, \pi_{R}\left(\left.\varphi\right|_{\Omega_{R}}\right)\right) ; X_{0}\left(\Omega_{R}\right)\right\| \\
& \leqq \| g\left(x, \pi_{R}\left(\left.\varphi\right|_{\Omega_{R}}\right) ; Y_{0} \|<\infty, \quad \forall \varphi \in X_{1} .\right.
\end{aligned}
$$


This proves part 1). By the same argument we have

$$
\begin{aligned}
& \left\|g\left(x, \varphi^{(1)}\right)-g\left(x, \varphi^{(2)}\right) ; X_{0}\left(\Omega_{R}\right)\right\| \\
& \leqq C(\varrho)\left\|\pi_{R}\left(\left.\varphi^{(1)}\right|_{\Omega_{R}}\right)-\pi_{R}\left(\left.\varphi^{(2)}\right|_{\Omega_{R}}\right) ; Y_{1}\right\| \\
& \leqq C(\varrho) d(R)\left\|\varphi^{(1)}-\varphi^{(2)} ; X_{1}\left(\Omega_{R}\right)\right\|,
\end{aligned}
$$

for all

$$
\varphi^{(k)} \in X_{1}, \quad\left\|\varphi^{(k)} ; X_{1}\left(\Omega_{R}\right)\right\| \leqq \varrho / d(R), \quad k=1,2 .
$$

This establishes part 2).

It is important to find conditions on the potential $U \in \mathscr{U}(X)$ and on the function $\varphi \in X_{1}$, which guarantee that $F_{\varphi} \in \mathscr{U}(Y)$ [see Eq. (1.5)].

Definition 3. Given $U \in \mathscr{U}(X)$, we say that $\varphi \in X_{1}$ is admissible with respect to $U$ if $F_{\varphi}$ belongs to $\mathscr{U}(Y)$.

The following two lemmas give sufficient conditions for admissibility.

Lemma 2. Let $U \in \mathscr{U}(X)$ and $\varphi \in X_{1}$ with the properties

1) $\varphi \in \bigoplus_{n} L^{\infty}\left(\mathbb{R}^{s}\right)$.

2) The map $\mathbb{R}^{s} \times Y_{1} \ni(x, \chi) \mapsto \nabla_{z} U(x, \varphi+\chi)$ is globally Lipschitz continuous.

3) For any compact subset $K \subset \mathbb{R}^{n}$ there exist non negative contants $a_{i}, i=1,2,3$, such that

$$
\begin{aligned}
& U(x, z) \leqq a_{1}, \quad\left|\nabla_{z} U(x, z)\right| \leqq a_{2}, \\
& \nabla_{z} \nabla_{z} U(x, z) \geqq-a_{3} \mathbb{1}_{\mathbb{R}^{n}}, \quad \forall(x, z) \in \mathbb{R}^{s} \times K .
\end{aligned}
$$

Then $\varphi$ is admissible with respect to $U$.

Proof. Conditions i)' and ii)' of Definition 2 are immediate consequences of hypothesis 2) and the identity

$$
\nabla_{z} F_{\varphi}(x, z)=\nabla_{z} U(x, \varphi(x)+z)-\nabla_{z} U(x, \varphi(x))
$$

To prove that Inequation (1.3)" is satisfied we first apply the mean value theorem

$$
F_{\varphi}(x, y)=\int_{0}^{1}(1-\sigma)\left\langle\nabla_{z} \nabla_{z} U(x, \varphi(x)+\sigma y) y, y\right\rangle d \sigma
$$

which yields, by hypotheses 1 and 3,

$$
F_{\varphi}(x, y) \geqq\left(-a_{3} / 2\right)|y|^{2}, \quad x \in \mathbb{R}^{s}, \quad|y| \leqq 1 .
$$

On the other hand, Equation (1.5) and Inequation (1.3)' imply

$$
F_{\varphi}(x, y) \geqq-\alpha-\beta|y+\varphi(x)|^{2}-U(x, \varphi(x))-\left\langle\nabla_{z} U(x, \varphi(x)), y\right\rangle
$$

which, for $|y| \geqq 1$, yields

$$
\begin{aligned}
F_{\varphi}(x, y) \geqq & -\left[\alpha+\beta+\beta \sup _{x \in \mathbb{R}^{s}}\left\{|\varphi(x)|^{2}+2|\varphi(x)|+\left|\nabla_{z} U(x, \varphi(x))\right|\right\}\right. \\
& \left.+\max \left(0, \sup _{x \in \mathbb{R}^{s}} U(x, \varphi(x))\right)\right]|y|^{2} .
\end{aligned}
$$


Lemma 3. Let $U \in \mathscr{U}(X)$ and $\varphi \in X_{1}$ with the following properties

1) Condition 2) of Lemma 2 holds

2) There exists a non negative constant $b$ such that

$$
\nabla_{z} \nabla_{z} U(x, z) \geqq-b \mathbb{1}_{\mathbb{R}^{n}}, \quad \forall(x, z) \in \mathbb{R}^{s} \times \mathbb{R}^{n} .
$$

Then $\varphi$ is admissible with respect to $U$.

Proof. By Lemma 2 only Inequation (1.3)" remains to be checked. This follows immediately by the mean value theorem, Equation (1.6), and the property 2).

Remark 1. Condition 2) of Lemma 2 is implied by the following condition on $U$ :

For any $\varrho>0$ there exists a positive constant $C(\varrho)$ such that

$$
\sup _{k=1, \ldots, n}\left\|\sum_{j=1}^{n} \frac{\partial^{2} U}{\partial z_{j} \partial z_{k}}\left(x, \chi^{(1)}+\varphi\right) \chi_{j}^{(2)} ; Y_{0}\right\| \leqq C(\varrho)\left\|\chi^{(2)} ; Y_{1}\right\|
$$

for all $\chi^{(k)} \in Y_{1}, k=1,2$, with $\left\|\chi^{(1)} ; Y_{1}\right\| \leqq \varrho$.

This is a trivial consequence of the mean value theorem.

Remark2. If $U \in \mathscr{U}(X)$ does not depend on the $x$ variables conditions 3) of Lemma 2 are automatically satisfied whereas condition 2) of Lemma 3 does not hold in general.

We now list some concrete interesting examples of interactions to which the above lemmas can be applied.

Proposition 1. Let $U(x, z): \mathbb{R}^{s} \times \mathbb{R}^{n} \rightarrow \mathbb{R}$ be a function satisfying condition iii) of Definition 2 and of the type listed below

$S=1$

$$
U(x, z)=\sum_{\alpha \in \mathbb{N}^{n}} c_{\alpha}(x) z^{\alpha}
$$

such that

1) $c_{\alpha} \in L^{\infty}\left(\mathbb{R}^{1}\right), \quad \forall \alpha$.

2) $\sum_{\alpha}\left\|c_{\alpha} ; L^{\infty}\left(\mathbb{R}^{1}\right)\right\| \sigma^{|\alpha|}<\infty, \quad \forall \sigma>0$.

$s=2$

$U(x, z)=\sum_{\alpha \in \mathbb{N}^{n}} c_{\alpha}(x) z^{\alpha}$ satisfying condition 1) as in the previous case and

$2)^{\prime} \sum_{\alpha}\left\|c_{\alpha} ; L^{\infty}\left(\mathbb{R}^{2}\right)\right\||\alpha|^{|\alpha| / 2} \sigma^{|\alpha|}<\infty, \quad \forall \sigma>0$. $s=3$

$U(x, z)$ is a real function of class $C^{(2)}$ in the $z$ variables with

1) $U(x, 0) \in L^{\infty}\left(\mathbb{R}^{3}\right), \nabla_{z} U(x, 0) \in \bigoplus L^{\infty}\left(\mathbb{R}^{3}\right)$.

2) There is a positive constant $\stackrel{n}{C}$ such that

$$
\sup _{x \in \mathbb{R}^{3}}\left\|\nabla_{z} \nabla_{z} U(x, z)\right\| \leqq C\left(1+|z|^{2}\right), \quad z \in \mathbb{R}^{n} .
$$


Then $U \in \mathscr{U}(X)$ and any $\varphi \in X_{1} \cap\left(\bigoplus_{n} L^{\infty}\left(\mathbb{R}^{s}\right)\right)$ is admissible with respect to $U$.

Proof. To prove both assertions it will be enough to establish that $U$ satisfies the estimate (1.7) for all $\varphi \in X_{1} \cap\left(\bigoplus_{n} L^{\infty}\left(\mathbb{R}^{s}\right)\right)$. In fact, by Remark 1 , estimate (1.7) implies that the map $\mathbb{R}^{s} \times Y_{1} \ni(x, \chi) \mapsto \nabla_{z} U(x, \varphi+\chi)$ is globally Lipschitz continuous. Then, by Lemma 1 (part 2), the map $\mathbb{R}^{s} \times X_{1} \ni(x, \theta) \mapsto \nabla_{z} U(x, \theta)$ is locally Lipschitz continuous. Moreover, since by hypothesis $\nabla_{z} U(x, 0) \in \bigoplus_{n} L^{\infty}\left(\mathbb{R}^{s}\right) \subset X_{0}$ the identity $\nabla_{z} U(x, \theta)=\left[\nabla_{z} U(x, \theta)-\nabla_{z} U(x, 0)\right]+\nabla_{z} U(x, 0)$ implies that condition i) of Definition 2 is also satisfied. This shows that $U \in \mathscr{U}(X)$. The admissibility of $\varphi$ follows from Lemma 2. The proof of estimate (1.7) is reduced to estimating in $Y_{0}$ terms of the type $\left(\varphi+\chi^{(1)}\right)^{\alpha} \chi^{(2)}$, with $\chi^{(k)} \in Y_{1}, k=1,2, \alpha \in \mathbb{N}^{n}$ for $s=1,2,|\alpha| \leqq 2$ for $s=3$. Now

$$
\begin{aligned}
\left\|\left(\varphi+\chi^{(1)}\right)^{\alpha} \chi^{(2)} ; Y_{0}\right\| \leqq & 2^{|\alpha|}\left\{\left\||\varphi|^{|\alpha|}\left|\chi^{(2)}\right| ; L^{2}\left(\mathbb{R}^{s}\right)\right\|\right. \\
& \left.+\left\|\left|\chi^{(1)}\right||\alpha|\left|\chi^{(2)}\right| ; L^{2}\left(\mathbb{R}^{s}\right)\right\|\right\} .
\end{aligned}
$$

The first term on the r.h.s. of Inequality (1.8) is immediately estimated by

$$
2^{|\alpha|}\left\||\varphi|^{|\alpha|}\left|\chi^{(2)}\right| ; L^{2}\left(\mathbb{R}^{s}\right)\right\| \leqq A^{|\alpha|}\left\|\varphi ; \bigoplus_{n} L^{\infty}\left(\mathbb{R}^{s}\right)\right\|^{|\alpha|}\left\|\chi^{(2)} ; Y_{1}\right\|
$$

We estimate the second term by applying the usual Sobolev inequalities ${ }^{2}$

$$
\begin{aligned}
& 2^{|\alpha|}\left\|\left|\chi^{(1)}\right|^{|\alpha|}\left|\chi^{(2)}\right| ; L^{2}\left(\mathbb{R}^{s}\right)\right\| \\
& \leqq 2^{|\alpha|}\left\|\left|\chi^{(1)}\right| ; L^{2(|\alpha|+1)}\left(\mathbb{R}^{s}\right)\right\|^{|\alpha|}\left\|\left|\chi^{(2)}\right| ; L^{2(|\alpha|+1)}\left(\mathbb{R}^{s}\right)\right\| \\
& \leqq\left\{B^{|\alpha|} C_{s}(2|\alpha|+2)^{|\alpha|+1}\left\|\chi^{(1)} ; Y_{1}\right\|^{|\alpha|}\right\}\left\|\chi^{(2)} ; Y_{1}\right\| .
\end{aligned}
$$

For $s=3$ the proof is completed. For $s=1,2$, the convergence of the sum over the $\alpha$ 's is taken care by conditions 2 ) and 2)' respectively.

Proposition 2. Let $U(x, z): \mathbb{R}^{s} \times \mathbb{R}^{n} \rightarrow \mathbb{R}$ be a function satisfying the hypotheses of Proposition 1 and such that there is a non negative constant $b$ for which

$$
\nabla_{z} \nabla_{z} U(x, z) \geqq-b \mathbb{1}_{\mathbb{R}^{n}}, \quad \forall(x, z) \in \mathbb{R}^{s} \times \mathbb{R}^{n} .
$$

Let $\varphi \in X_{1}$ be such that for some cube $Q$ of size $R$

$$
\sup _{m \in \mathbb{Z}^{s}}\left\|\varphi ; X_{1}(Q+R m)\right\|<\infty,
$$

then $U \in \mathscr{U}(X)$ and $\varphi$ is admissible with respect to $U$.

2 For the convenience of the reader we recall the Sobolev inequalities [8]:

$$
\begin{array}{lll}
s=1\left\|f ; L^{p}\left(\mathbb{R}^{1}\right)\right\| \leqq C_{1}(p)\left\|f ; H^{1}\left(\mathbb{R}^{1}\right)\right\|, & 2 \leqq p \leqq \infty, & C_{1}(p)=0(1), \\
s=2\left\|f ; L^{p}\left(\mathbb{R}^{2}\right)\right\| \leqq C_{2}(p)\left\|f ; H^{1}\left(\mathbb{R}^{2}\right)\right\|, & 2 \leqq p<\infty, & C_{2}(p)=0\left(p^{1 / 2}\right), \\
s=3\left\|f ; L^{p}\left(\mathbb{R}^{3}\right)\right\| \leqq C_{3}(p)\left\|f ; H^{1}\left(\mathbb{R}^{3}\right)\right\|, & 2 \leqq p \leqq 6, & C_{3}(p)=0(1)
\end{array}
$$

The same kind of estimates hold locally. In particular, for any cube $k \subset \mathbb{R}^{s}$ of size $R$, they take the form

$\left\|f ; L^{p}(k)\right\| \leqq C_{s, R}(p)\left\|f ; H^{1}(k)\right\|$,

with $p \in[2,+\infty]$ for $s=1, p \in\left[2,+\infty\left[\right.\right.$ for $s=2$ and $p \in[2,6]$ for $s=3$. The constants $C_{s, R}(p)$ depend only on the size $R$ and exhibit the same dependence on $p$ as in the global case 
Proof. As remarked at the beginning of the proof of Proposition 1, to establish that $U \in \mathscr{U}(X)$ it is enough to prove that $U$ satisfies the estimate (1.7) [for $\varphi$ satisfying $\left.(1.7)^{\prime}\right]$. The admissibility of $\varphi$ then follows from Lemma 3 . Actually by Inequation (1.8) we need to estimate only the term $\left\||\varphi|^{|\alpha|}\left|\chi^{(2)}\right| ; L^{2}\left(\mathbb{R}^{s}\right)\right\|$. By successive applications of Hölder and local Sobolev inequalities ${ }^{2}$ one obtains

$$
\begin{aligned}
\left\||\varphi|^{|\alpha|}\left|\chi^{(2)}\right| ; L^{2}\left(\mathbb{R}^{s}\right)\right\|^{2} \leqq & \sum_{m \in \mathbb{Z}^{s}}\left(\int_{Q+R m}|\varphi|^{2(|\alpha|+1)} d x\right)^{|\alpha| \mid(|\alpha|+1)} \\
& \cdot\left(\int_{Q+R m}\left|\chi^{(2)}\right|^{2(|\alpha|+1)} d x\right)^{1 /(|\alpha|+1)} \\
\leqq & \sum_{m \in \mathbb{Z}^{s}} B^{2|\alpha|+2} C_{s, R}(2|\alpha|+2)^{2|\alpha|+2} \\
& \cdot\left\|\varphi ; X_{1}(Q+R m)\right\|^{2|\alpha|}\left\|\chi^{(2)} ; X_{1}(Q+R m)\right\|^{2} \\
\leqq & 2^{s}\left\{\left(\sup _{m \in \mathbb{Z}^{s}}\left\|\varphi ; X_{1}(Q+R m)\right\|\right)^{2|\alpha|}\right. \\
& \left.\cdot B^{2|\alpha|+2} C_{s, R}(2|\alpha|+2)^{2|\alpha|+2}\right\}\left\|\chi^{(2)} ; Y_{1}\right\|^{2} .
\end{aligned}
$$

\section{Hilbert Space Sectors-Stability and Time Evolution}

We already know (Section 1, Theorems 1 and 2) that for any $U \in \mathscr{U}(X)$ the integral Equation (1.2) has a unique $C^{(0)}(\mathbb{R}, X)$-solution with initial data in $X$. It is convenient to denote by $\mathscr{F}_{U}, U \in \mathscr{U}(X)$, the set of all such solutions. There is a canonical map from $X$ onto $\mathscr{F}_{U}$, namely the map which to every $\left(\begin{array}{l}\varphi \\ \psi\end{array}\right) \in X$ associates the solution $\left(\begin{array}{l}\varphi(t) \\ \psi(t)\end{array}\right) \in \mathscr{F}_{U}$ with $\left(\begin{array}{l}\varphi(0) \\ \psi(0)\end{array}\right)=\left(\begin{array}{l}\varphi \\ \psi\end{array}\right)$. As already motivated in the introduction we partition $\mathscr{F}_{U}$ by the following equivalence relation.

Definition 4. On $\mathscr{F}_{U}$ we define the relation

$$
\left(\begin{array}{l}
\varphi(t) \\
\psi(t)
\end{array}\right) \sim\left(\begin{array}{l}
\varphi^{\prime}(t) \\
\psi^{\prime}(t)
\end{array}\right) \Leftrightarrow\left(\begin{array}{l}
\varphi(t)-\varphi^{\prime}(t) \\
\psi(t)-\psi^{\prime}(t)
\end{array}\right) \in C^{(0)}(\mathbb{R}, Y)
$$

The quotient set will be denoted by $\mathscr{F}_{U}^{\sim}$.

Relation (2.1) can be interpreted by saying that $\left(\begin{array}{l}\varphi^{\prime}(t) \\ \psi^{\prime}(t)\end{array}\right)$ is, for all $t \in \mathbb{R}$, a "small" perturbation of $\left(\begin{array}{l}\varphi(t) \\ \psi(t)\end{array}\right)$. The partition of $\mathscr{F}_{U}$ into classes of equivalence induces in an obvious way a partition of $X$. However, we are mainly interested in those classes of $X$ which are left invariant under time evolution. This leads naturally to the following

Definition 5. By $Z$ we will denote the set of all $\left(\begin{array}{l}\varphi \\ \psi\end{array}\right) \in X$ such that

$$
\left(\begin{array}{c}
\varphi(t)-\varphi \\
\psi(t)-\psi
\end{array}\right) \in C^{(0)}(\mathbb{R}, Y)
$$


In $Z$ we introduce the equivalence relation

$$
\left(\begin{array}{c}
\varphi \\
\psi
\end{array}\right) \approx\left(\begin{array}{c}
\varphi^{\prime} \\
\psi^{\prime}
\end{array}\right) \Leftrightarrow\left(\begin{array}{c}
\varphi-\varphi^{\prime} \\
\psi-\psi^{\prime}
\end{array}\right) \in Y
$$

The quotient set will be denoted by $Z^{\sim}$. Each element of $Z^{\sim}$ will be called a Hilbert space sector in, $X$. By $\mathscr{H}_{(\varphi, \varphi)}$ we denote the Hilbert space sector containing $\left(\begin{array}{c}\varphi \\ \psi\end{array}\right)$

It is clear that $Z$ does not depend on the choice of the initial time $t=0$ and that the canonical map from $Z$ into $\mathscr{F}_{U}$ induces an injective map from $Z^{\sim}$ into $\mathscr{F}_{U}^{\sim}$.

In the next theorem we will find, under suitable assumptions, a necessary condition for an element $\left(\begin{array}{l}\varphi_{0} \\ \psi_{0}\end{array}\right) \in X$ to belong to $Z$. For this purpose it is convenient to establish the following technical

Lemma 4. For all $h, \psi \in \mathscr{D}^{\prime}\left(\mathbb{R}^{s}\right)$, the map

$$
\mathbb{R} \ni t \mapsto\left(\begin{array}{c}
(-\Delta)^{-1}\left(1-\cos \left((-\Delta)^{1 / 2} t\right)\right) h+(-\Delta)^{-1 / 2} \sin \left((-\Delta)^{1 / 2} t\right) \psi \\
(-\Delta)^{-1 / 2} \sin \left((-\Delta)^{1 / 2} t\right) h+\left(\cos \left((-\Delta)^{1 / 2} t\right)-1\right) \psi
\end{array}\right) \equiv\left(\begin{array}{c}
A(t) \\
B(t)
\end{array}\right)
$$

belongs to $C^{(0)}\left(\mathbb{R}, H^{1}\left(\mathbb{R}^{s}\right) \oplus L^{2}\left(\mathbb{R}^{s}\right)\right)$ if and only if $h \in H^{-1}\left(\mathbb{R}^{s}\right)$ and $\psi \in L^{2}\left(\mathbb{R}^{s}\right)$.

Proof. Expression (2.3) must be understood in the following sense

$$
\begin{aligned}
& A(t)=\mathscr{F}^{-1}\left(|\xi|^{-2}(1-\cos |\xi| t)(\mathscr{F} h)(\xi)+|\xi|^{-1} \sin |\xi| t(\mathscr{F} \psi)(\xi)\right) \\
& B(t)=\mathscr{F}^{-1}\left(|\xi|^{-1} \sin |\xi| t(\mathscr{F} h)(\xi)+(\cos |\xi| t-1)(\mathscr{F} \psi)(\xi)\right) .
\end{aligned}
$$

$A(t)$ and $B(t)$ are well defined elements of $\mathscr{D}^{\prime}\left(\mathbb{R}^{s}\right)$ since the coefficients of $(\mathscr{F} h)(\xi)$ and of $(\mathscr{F} \psi)(\xi)$ are analytic functions of $|\xi|^{2}$ bounded in the complex space $\mathbb{C}^{s}$ by const $\exp ($ const $|\operatorname{Im} z|)$, as can be seen by application of a Phragmen-Lindelöf Theorem [9]. If $h \in H^{-1}\left(\mathbb{R}^{s}\right)$ and $\varphi \in L^{2}\left(\mathbb{R}^{s}\right)$, to see that the map (2.3) belongs to $C^{(0)}\left(\mathbb{R}, H^{1}\left(\mathbb{R}^{s}\right) \oplus L^{2}\left(\mathbb{R}^{s}\right)\right)$ it is enough to prove that the maps

$$
\mathbb{R} \ni \mapsto\left\{\begin{array}{l}
(1+\sqrt{-\Delta})^{2}(-\Delta)^{-1}(\cos \sqrt{-\Delta} t-1) \\
(1+\sqrt{-\Delta})(-\Delta)^{-1 / 2}(\sin \sqrt{-\Delta} t) \\
\cos \sqrt{-\Delta} t-1
\end{array}\right.
$$

belong to $C^{(0)}\left(\mathbb{R}, \mathscr{L}\left(L^{2}\left(\mathbb{R}^{s}\right), L^{2}\left(\mathbb{R}^{s}\right)\right)\right)$, with the strong topology of $\mathscr{L}\left(L^{2}\left(\mathbb{R}^{s}\right), L^{2}\left(\mathbb{R}^{s}\right)\right)$. The proof is trivial and will be omitted. Conversely, by the continuity of the map (2.3) we can integrate $B(t)$ and obtain

$$
\int_{0}^{t} B(\tau) d \tau=\left((-\Delta)^{-1}(1-\cos \sqrt{-\Delta} t) h+(-\Delta)^{-1 / 2}(\sin \sqrt{-\Delta} t) \psi-t \psi\right) \in L^{2}\left(\mathbb{R}^{s}\right) .
$$

By comparison with the ex pression for $A(t)$ it follows that $\psi \in L^{2}\left(\mathbb{R}^{s}\right)$. Consequently,

$$
(-\Delta)^{-1 / 2} \sin \left((-\Delta)^{1 / 2}\right) \psi \in C^{(0)}\left(\mathbb{R}, H^{1}\left(\mathbb{R}^{s}\right)\right)
$$

and therefore

$$
(-\Delta)^{-1}\left(1-\cos \left((-\Delta)^{1 / 2} t\right)\right) h \in C^{(0)}\left(\mathbb{R}, H^{1}\left(\mathbb{R}^{s}\right)\right) .
$$


Finally, $h \in H^{-1}\left(\mathbb{R}^{s}\right)$ if we prove that

$$
\int_{|\xi| \leqq 2}|(\mathscr{F} h)(\xi)|^{2} d \xi+\int_{|\xi| \geqq 2}|\xi|^{-2}|(\mathscr{F} h)(\xi)|^{2} d \xi \equiv I_{1}+I_{2}<\infty .
$$

The integral $I_{1}$ is taken care by the estimate

$$
|\xi|^{-2}(\cos |\xi| t-1)|(\mathscr{F} h)(\xi)| \geqq \frac{t^{2}}{4}|(\mathscr{F} h)(\xi)|
$$

which is valid for $|\xi| \leqq 2$ and $t$ sufficiently small. On the other hand, integration of the $L^{2}$-valued continuous function $(-\Delta)^{-1 / 2}\left(\cos (-\Delta)^{1 / 2} t-1\right) h$ yields $\left((-\Delta)^{-1} \sin (-\Delta)^{1 / 2}-(-\Delta)^{-1 / 2}\right) h \in L^{2}\left(\mathbb{R}^{s}\right)$. Now the finiteness of the integral $I_{2}$ is a consequence of the estimate

$$
\left(|\xi|^{-2} \sin |\xi|-|\xi|^{-1}\right)|(\mathscr{F} h)(\xi)| \geqq \frac{1}{2}|\xi|^{-1}|(\mathscr{F} h)(\xi)|
$$

which is valid for $|\xi| \geqq 2$.

Theorem 3. Let $M(x, z): \mathbb{R}^{s} \times \mathbb{R}^{n} \rightarrow \mathbb{R}^{n}$ be a function such that $X_{1} \ni \lambda \mapsto M(x, \lambda(x))$ is a continuous $X_{0}$-valued map. Let $\left(\begin{array}{l}\varphi(t) \\ \psi(t)\end{array}\right) \in C^{(0)}(\mathbb{R}, X)$ be a solution of the integral equation

$$
\left(\begin{array}{l}
\varphi(t) \\
\psi(t)
\end{array}\right)=W(t)\left(\begin{array}{l}
\varphi_{0} \\
\psi_{0}
\end{array}\right)+\int_{0}^{t} W(t-s)\left(\begin{array}{c}
0 \\
-M(x, \varphi(s))
\end{array}\right) d s
$$

with $\left(\begin{array}{c}\varphi_{0} \\ \psi_{0}\end{array}\right) \in X$.

If

a) $Y_{1} \ni \chi \mapsto M\left(x, \varphi_{0}+\chi\right)-M\left(x, \varphi_{0}\right)$ is a continuous $Y_{0}$-valued map;

b) $\left(\begin{array}{c}\varphi(t)-\varphi_{0} \\ \psi(t)-\psi_{0}\end{array}\right) \in C^{(0)}(\mathbb{R}, Y)$;

then

$$
\left\{\begin{array}{l}
\Delta \varphi_{0}-M\left(x, \varphi_{0}\right) \equiv h \in \bigoplus_{n} H^{-1}\left(\mathbb{R}^{s}\right), \\
\psi_{0} \in Y_{0}
\end{array}\right.
$$

Proof. It is convenient to recall that

$$
\begin{aligned}
W(t) & =\left(\begin{array}{cc}
\cos \left((-\Delta)^{1 / 2} t\right) & (-\Delta)^{-1 / 2} \sin \left((-\Delta)^{1 / 2} t\right) \\
-(-\Delta)^{1 / 2} \sin \left((-\Delta)^{1 / 2} t\right) & \cos \left((-\Delta)^{1 / 2} t\right)
\end{array}\right) \otimes \mathbb{1}_{\mathbb{C}^{n}} \\
& \equiv\left[\begin{array}{cc}
\mathscr{F}^{-1} \circ\left(\begin{array}{cc}
\cos |\xi| t & |\xi|^{-1} \sin |\xi| t \\
-|\xi| \sin |\xi| t & \cos |\xi| t
\end{array}\right) \circ \mathscr{F}
\end{array}\right] \otimes \mathbb{1}_{\mathbb{C}^{n}} .
\end{aligned}
$$

Then, it can be checked by direct computation that the quantities

$$
\begin{aligned}
& \chi(t) \equiv \varphi(t)-\varphi_{0} \\
& \zeta(t) \equiv \psi(t)-\psi_{0}
\end{aligned}
$$


satisfy the following integral equation

$$
\begin{aligned}
\left(\begin{array}{l}
\chi(t) \\
\zeta(t)
\end{array}\right) & =\left[\left(\begin{array}{cc}
(-\Delta)^{-1}\left(1-\cos \left((-\Delta)^{1 / 2} t\right)\right) & (-\Delta)^{-1 / 2} \sin \left((-\Delta)^{1 / 2} t\right) \\
(-\Delta)^{-1 / 2} \sin \left((-\Delta)^{1 / 2} t\right) & \cos \left((-\Delta)^{1 / 2} t\right)-1
\end{array}\right) \otimes \mathbb{1}_{\mathbb{C}^{n}}\right]\left(\begin{array}{c}
h \\
\psi_{0}
\end{array}\right) \\
& +\int_{0}^{t} W(t-s)\left(\begin{array}{cc}
0 \\
-M\left(x, \varphi_{0}+\chi(s)\right)+M\left(x, \varphi_{0}\right)
\end{array}\right) d s .
\end{aligned}
$$

The assertion is now an immediate consequence of conditions a), b) and of Lemma 4. Remark 3. If $U \in \mathscr{U}(X)$ and $\varphi_{0}$ is admissible with respect to $U$ then Theorem 3 holds once $M(x, z)$ is identified with $\nabla_{z} U$.

The next fundamental theorem, together with Lemmas 2, 3 and Propositions 1,2 , guarantees the existence of non trivial Hilbert space sectors.

Theorem 4. Let $U \in \mathscr{U}(X)$ and let $\left(\begin{array}{l}\varphi_{0} \\ \psi_{0}\end{array}\right) \in X$ such that

a) $h \equiv \Delta \varphi_{0}-\nabla_{z} U\left(x, \varphi_{0}\right) \in \bigoplus_{n} H^{-1}\left(\mathbb{R}^{s}\right), \quad \psi_{0} \in Y_{0}$.

b) $\varphi_{0}$ is admissible with respect to $U$.

Then $\left(\begin{array}{l}\varphi_{0} \\ \psi_{0}\end{array}\right) \in Z$ (see Definition 5) and

$$
\mathscr{H}_{\left(\varphi_{0}, \psi_{0}\right)}=\left\{\left(\begin{array}{l}
\varphi \\
\psi
\end{array}\right) \in X \mid\left(\begin{array}{c}
\varphi-\varphi_{0} \\
\psi-\psi_{0}
\end{array}\right) \in Y\right\}=\left(\begin{array}{c}
\varphi_{0} \\
\psi_{0}
\end{array}\right)+Y .
$$

Proof. In the same way as in the proof of Theorem 3 we consider the following integral equation

$$
\left(\begin{array}{l}
\chi(t) \\
\zeta(t)
\end{array}\right)=W(t)\left(\begin{array}{c}
\chi_{0} \\
\zeta_{0}
\end{array}\right)+L(t)+\int_{0}^{t} W(t-s)\left(\begin{array}{c}
0 \\
-\nabla_{z} F_{\varphi_{0}}(x, \chi(s))
\end{array}\right) d s
$$

where

$$
L(t)=\left[\left(\begin{array}{cc}
(-\Delta)^{-1}\left(1-\cos \left((-\Delta)^{1 / 2} t\right)\right) & (-\Delta)^{-1 / 2} \sin \left((-\Delta)^{1 / 2} t\right) \\
(-\Delta)^{-1 / 2} \sin \left((-\Delta)^{1 / 2} t\right) & \cos \left((-\Delta)^{1 / 2} t\right)-1
\end{array}\right) \otimes \mathbb{1}_{\mathbb{C}^{n}}\right]\left(\begin{array}{c}
h \\
\psi_{0}
\end{array}\right) .
$$

By condition a) and Lemma $4, t \mapsto L(t)$ is a continuous $Y$-valued map with $L(0)=0$. Furthermore, $W(t)$ is a one-parameter strongly continuous group of linear bounded operators in $Y$. Therefore, it makes sense to consider Equation (2.12) in $Y$. It is important to remark that if $\left(\begin{array}{l}\chi(t) \\ \zeta(t)\end{array}\right)$ is any continuous $Y$-valued solution of Equation (2.12), with $\left(\begin{array}{c}\chi_{0} \\ \zeta_{0}\end{array}\right) \in Y$, then $\left(\begin{array}{c}\chi(t)+\varphi_{0} \\ \zeta(t)+\psi_{0}\end{array}\right)$ is a continuous $X$-valued solution of Equation (1.2) with initial data $\left(\begin{array}{l}\varphi_{0}+\chi_{0} \\ \psi_{0}+\zeta_{0}\end{array}\right)$ as can be seen by direct inspection. The theorem now follows easily if we admit for the moment that Equation (2.12) has a unique solution $\left(\begin{array}{l}\chi(t) \\ \zeta(t)\end{array}\right) \in C^{(0)}(\mathbb{R}, Y)$ for any $\left(\begin{array}{c}\chi_{0} \\ \zeta_{0}\end{array}\right) \in Y$. Let $\left(\begin{array}{l}\varphi(t) \\ \psi(t)\end{array}\right) \in C^{(0)}(\mathbb{R}, X)$ 
be the solution of Equation (1.2) with initial data $\left(\begin{array}{c}\varphi_{0} \\ \psi_{0}\end{array}\right)$ and let $\left(\begin{array}{l}\chi(t) \\ \zeta(t)\end{array}\right) \in C^{(0)}(\mathbb{R}, Y)$ be the solution of Equation (2.12) with zero initial data. Theorem 1 implies that $\varphi(t)=\chi(t)+\varphi_{0}, \psi(t)=\zeta(t)+\psi_{0}$, and therefore that $\left(\begin{array}{c}\varphi_{0} \\ \psi_{0}\end{array}\right) \in Z$. Let now $\left(\begin{array}{c}\alpha(t) \\ \beta(t)\end{array}\right) \in C^{(0)}$ $(\mathbb{R}, X)$ be the solution of Equation (1.2) with initial data $\left(\begin{array}{c}\alpha_{0} \\ \beta_{0}\end{array}\right)$ such that $\left(\begin{array}{c}\varphi_{0}-\alpha_{0} \\ \psi_{0}-\beta_{0}\end{array}\right) \in Y$. If we denote by $\left(\begin{array}{l}\varrho(t) \\ \sigma(t)\end{array}\right) \in C^{(0)}(\mathbb{R}, Y)$ the solution of Equation (2.12) with data $\varrho_{0}=\alpha_{0}-\varphi_{0}, \sigma_{0}=\beta_{0}-\psi_{0}$, then, by Theorem $1, \varrho(t)+\varphi_{0}=\alpha(t), \sigma(t)+\psi_{0}=\beta(t)$, and therefore $\left(\begin{array}{c}\alpha_{0} \\ \beta_{0}\end{array}\right) \in \mathscr{H}_{\left(\varphi_{0}, \psi_{0}\right)}$.

To prove that Equation (2.12) has a unique solution $\left(\begin{array}{l}\chi(t) \\ \zeta(t)\end{array}\right) \in C^{(0)}(\mathbb{R}, Y)$ for any initial data $\left(\begin{array}{l}\chi_{0} \\ \zeta_{0}\end{array}\right) \in Y$ we apply Theorem A of Appendix A. The only non trivial hypothesis to be verified is Inequality (A.3) that we are going to establish from energy conservation and from the lower bounds of the potential. To express the energy conservation it is convenient to work with the differential equation associated to the integral Equation (2.12). For this purpose, to avoid domain problems, let us define the linear operators

$$
\begin{aligned}
& P_{r} \equiv\left(\mathscr{F}^{-1} \circ p_{r}(\xi) \circ \mathscr{F}\right) \otimes \mathbb{1}_{\mathbb{C}^{n}}, \quad r>0, \\
& P_{r}(\xi)= \begin{cases}1, & |\xi| \leqq r \\
0, & |\xi|>r .\end{cases}
\end{aligned}
$$

It is trivial to verify that

$$
\begin{aligned}
& {\left[\begin{array}{cc}
P_{r} & 0 \\
0 & P_{r}
\end{array}\right] W(t)=W(t)\left[\begin{array}{cc}
P_{r} & 0 \\
0 & P_{r}
\end{array}\right],} \\
& P_{r} f \in \bigcap_{m}\left(\bigoplus_{n} H^{m}\left(\mathbb{R}^{s}\right)\right), \quad \forall f \in \bigcup_{m}\left(\bigoplus_{n} H^{m}\left(\mathbb{R}^{s}\right)\right) .
\end{aligned}
$$

Now, application of the operators $\left[\begin{array}{cc}P_{r} & 0 \\ 0 & P_{r}\end{array}\right]$ to Equation (2.12) allows to differentiate in the time variable, obtaining

$$
\begin{aligned}
& \frac{d}{d t}\left(P_{r} \chi\right)=P_{r}\left(\zeta+\psi_{0}\right), \\
& \frac{d}{d t}\left(P_{r} \zeta\right)=\Delta P_{r} \chi+P_{r} h-P_{r} \nabla_{z} F_{\varphi_{0}}(\chi, \chi) .
\end{aligned}
$$

By a well known argument Equations (2.16) imply

$$
\begin{aligned}
& \frac{1}{2}\left[\left\langle P_{r} \nabla \chi(t), P_{r} \nabla \chi(t)\right\rangle+\left\langle P_{r}\left(\zeta+\psi_{0}\right)(t), P_{r}\left(\zeta+\psi_{0}\right)(t)\right\rangle-\left\langle P_{r} \chi(t), P_{r} h\right\rangle\right. \\
& -\frac{1}{2}\left[\left\langle P_{r} \nabla \chi(0), P_{r} \nabla \chi(0)\right\rangle+\left\langle P_{r}\left(\zeta+\psi_{0}\right)(0), P_{r}\left(\zeta+\psi_{0}\right)(0)\right\rangle\right]+\left\langle P_{r} \chi(0), P_{r} h\right\rangle \\
& =-\int_{0}^{t}\left\langle P_{r}\left(\zeta+\psi_{0}\right)(s), P_{r} \nabla_{z} F_{\varphi_{0}}(x, \chi(s))\right\rangle d s .
\end{aligned}
$$


By letting $r \rightarrow+\infty$ we obtain a relation exactly equal to $(2.17)$ in which $P_{r}$ is substituted by the identity.

Now, it can be verified (see Appendix B) that $\chi(t)$ is $L^{2}$-differentiable with $d \chi / d t=\zeta+\psi_{0}$, that $F_{\varphi_{0}}(x, \chi(t, x)) \in L^{1}\left(\mathbb{R}^{s}\right)$ for all $t$ and that

$$
\begin{aligned}
\int_{0}^{t}\left\langle\zeta(s)+\psi_{0}, \nabla_{z} F_{\varphi_{0}}(x, \chi(s))\right\rangle d s= & \int_{\mathbb{R}^{s}} F_{\varphi_{0}}(x, \chi(t, x)) d x \\
& -\int_{\mathbb{R}^{s}} F_{\varphi_{0}}(x, \chi(0, x)) d x .
\end{aligned}
$$

Equations (2.17) and (2.18) yield $\frac{d}{d t} E(t)=0$, where

$$
\begin{aligned}
E(t)= & \frac{1}{2}\left[\langle\nabla \chi(t), \nabla \chi(t)\rangle+\left\langle\zeta(t)+\psi_{0}, \zeta(t)+\psi_{0}\right\rangle\right] \\
& -\langle\chi(t), h\rangle+\int_{\mathbb{R}^{s}} F_{\varphi_{0}}(x, \chi(t, x)) d x .
\end{aligned}
$$

If we define

$$
H(t)=E(t)+\left(\gamma+\frac{1}{2}\right)\langle\chi(t), \chi(t)\rangle+\frac{1}{2}\left\langle\psi_{0}, \psi_{0}\right\rangle+\left\|h ; \underset{n}{\bigoplus_{n}} H^{-1}\left(\mathbb{R}^{s}\right)\right\|^{2},
$$

where $\gamma$ is the constant in Inequality (1.3)", it follows that

$$
H(t) \geqq \frac{1}{4}[\langle\chi(t), \chi(t)\rangle+\langle\zeta(t), \zeta(t)\rangle+\langle\nabla \chi(t), \nabla \chi(t)\rangle]
$$

and that

$$
d H(t) / d t=2\left(\gamma+\frac{1}{2}\right)\left[\langle\chi(t), \chi(t)\rangle+\left\langle\chi(t), \psi_{0}\right\rangle\right] .
$$

Finally, application of Gronwall's lemma yields

$$
\langle\chi(t), \chi(t)\rangle+\langle\nabla \chi(t), \nabla \chi(t)\rangle+\langle\zeta(t), \zeta(t)\rangle \leqq C_{1} e^{C_{2}|t|}
$$

which is the required a priori estimate to apply Theorem A of Appendix A.

\section{Total Energy and Momentum}

In the theory described by Equation (1.2) we take as expression for the energy density the following quantity

$$
K(\varphi, \psi)=\frac{1}{2} \sum_{j=1}^{n}\left(\left|\nabla \varphi_{j}\right|^{2}+\psi_{j}^{2}\right)+U(x, \varphi)
$$

which integrated over the whole space $\mathbb{R}^{s}$ is expected to yield the total energy. Unfortunately, if $\left(\begin{array}{l}\varphi \\ \psi\end{array}\right) \in X$ and $U \in \mathscr{U}(X)$, the function $K(\varphi, \psi)$ is locally but, in general, not globally integrable. However, keeping in mind that what one usually measures are energy differences, we would hope that for some pairs of elements of $X$ the difference of the corresponding energy densities is globally integrable. Natural candidates for the families of elements of $X$ having relatively finite total energy are the Hilbert space sectors defined in Section 2. 
Theorem 5. Let $\left(\begin{array}{l}\varphi \\ \psi\end{array}\right) \in X, U \in \mathscr{U}(X)$ such that
a) $h \equiv \Delta \varphi-\nabla_{z} U(x, \varphi) \in \bigoplus_{n} H^{-1}\left(\mathbb{R}^{s}\right)$ $\psi \in Y_{0}$.
b) $\varphi$ is admissible with respect to $U$.

Then, for all $\left(\begin{array}{c}\varphi^{\prime} \\ \psi^{\prime}\end{array}\right) \in \mathscr{H}_{(\varphi, \psi)}$ with $\operatorname{supp}\left(\varphi^{\prime}-\varphi\right)$ compact, the function $K\left(\varphi^{\prime}, \psi^{\prime}\right)-$ $H(\varphi, \psi) \in L^{1}\left(\mathbb{R}^{s}\right)$ and the functional

$$
\begin{aligned}
& \mathscr{H}_{(\varphi, \psi)} \ni\left(\begin{array}{c}
\varphi^{\prime} \\
\psi^{\prime}
\end{array}\right) \mapsto E_{(\varphi, \psi)}\left(\varphi^{\prime}, \psi^{\prime}\right) \\
& \equiv \int_{\mathbb{R}^{s}}\left[\left(K\left(\varphi^{\prime}, \psi^{\prime}\right)-K(\varphi, \psi)\right] d x, \quad \operatorname{supp}\left(\varphi^{\prime}-\varphi\right)\right. \text { compact }
\end{aligned}
$$

has a unique extension to the whole $\mathscr{H}_{(\varphi, \psi)}$ (which we will continue to denote by the same symbol).

Proof. A trivial computation yields

$$
\begin{aligned}
& K\left(\varphi^{\prime}, \psi^{\prime}\right)-K(\varphi, \psi) \\
& =V_{(\varphi, \psi)}(\chi, \zeta)+\sum_{j=1}^{n} \frac{\partial U}{\partial z_{j}}(x, \varphi) \chi_{j}+\sum_{j=1}^{n} \sum_{i=1}^{s} \frac{\partial \chi_{j}}{\partial x_{i}} \frac{\partial \varphi_{j}}{\partial x_{i}}
\end{aligned}
$$

where

$$
\begin{aligned}
& \chi \equiv \varphi^{\prime}-\varphi, \quad \zeta \equiv \psi^{\prime}-\psi, \\
& V_{(\varphi, \psi)}(\chi, \zeta) \equiv \frac{1}{2} \sum_{j=1}^{n}\left(\left|\nabla \chi_{j}\right|^{2}+\left(\zeta_{j}+\psi_{j}\right)^{2}-\psi_{j}^{2}\right)+F_{\varphi}(x, \chi) .
\end{aligned}
$$

Since $F_{\varphi}(x, \chi) \in L^{1}\left(\mathbb{R}^{s}\right)$ [see Eq. (B.3) of Appendix B] it follows that $V_{(\varphi, \psi)}(\chi, \zeta) \in$ $L^{1}\left(\mathbb{R}^{s}\right)$. Moreover the other two terms on the r.h.s. of Equation (3.3) belong to $L^{1}\left(\mathbb{R}^{s}\right)$ if $\chi$ is compactly supported. Therefore

$$
E_{(\varphi, \psi)}\left(\varphi^{\prime}, \psi^{\prime}\right)=\int_{\mathbb{R}^{s}} V_{(\varphi, \psi)}(\chi, \zeta) d x+L_{\varphi}(\chi)
$$

where

$$
L_{\varphi}(\chi) \equiv\left\langle\nabla_{z} U(x, \varphi), \chi\right\rangle_{H_{1 \mathrm{oc}}^{-1}, H_{\mathrm{comp}}^{1}}+\langle\nabla \varphi, \nabla \chi\rangle_{L_{\mathrm{loc}}^{2}, L_{\mathrm{comp}}^{2}}
$$

for $\chi$ with compact support.

The distribution $L_{\varphi}$ can obviously be rewritten as

$$
L_{\varphi}(\chi)=-\langle h, \chi\rangle_{H_{1 \mathrm{oc}}^{-1}, H_{\mathrm{comp}}^{1}}
$$

which, by hypothesis a), can be uniquely extended to all $\chi \in Y_{1}$. To complete the argument it will be enough to show that the map

$$
Y \ni\left(\begin{array}{l}
\varphi \\
\zeta
\end{array}\right) \mapsto V_{(\varphi, \psi)}(\chi, \zeta) \in L^{1}\left(\mathbb{R}^{s}\right)
$$


is continuous, i.e. that the map $Y_{1} \ni \chi \mapsto F_{\varphi}(x, \chi) \in L^{1}\left(\mathbb{R}^{s}\right)$ is continuous. This is a consequence of the following estimate based on the mean value theorem

$$
\begin{aligned}
& \left\|F_{\varphi}\left(x, \chi_{1}\right)-F_{\varphi}\left(x, \chi_{2}\right) ; L^{1}\left(\mathbb{R}^{s}\right)\right\| \\
& \leqq \sup _{0 \leqq \sigma \leqq 1}\left\|\nabla_{z} F_{\varphi}\left(x, \chi_{1}+\sigma\left(\chi_{2}-\chi_{1}\right)\right) ; Y_{0}\right\|\left\|\chi_{2}-\chi_{1} ; Y_{0}\right\|
\end{aligned}
$$

and of the fact that $F_{\varphi} \in \mathscr{U}(Y)$ by the admissibility of $\varphi$.

Remark 4. By Theorem 4 the Hilbert space sector $\mathscr{H}_{(\varphi, \psi)}$ is the affine variety $\left(\begin{array}{l}\varphi \\ \psi\end{array}\right)+Y$ (and thus isomorphic to $Y$ ). It is clear from the proof of Theorem 5 that the functional $E_{(\varphi, \psi)}$ is continuous on $\mathscr{H}_{(\varphi, \varphi)}$ equipped with the $Y$-topology.

Corollary 1. Let the hypotheses of Theorem 5 be satisfied and let $\left(\begin{array}{l}\varphi^{\prime} \\ \psi^{\prime}\end{array}\right) \in \mathscr{H}_{(\varphi, \psi)}$. If $\left(\begin{array}{l}\varphi^{\prime} \\ \psi^{\prime}\end{array}\right) \in C^{(0)}(\mathbb{R} ; X)$ is the solution of Equation (1.2) with initial data $\varphi^{\prime}(0)=\varphi^{\prime}$, $\psi^{\prime}(0)=\psi^{\prime}$, then

$$
E_{(\varphi, \psi)}\left(\varphi^{\prime}, \psi^{\prime}\right)=E_{(\varphi, \psi)}\left(\varphi^{\prime}(t), \psi^{\prime}(t)\right), \quad \forall t \in \mathbb{R} .
$$

Proof. Obvious by Theorem 4 [see ex pression (2.19)].

Since the energy measurements we are able to perform are always local in space, it should be possible to consider the energy functional $E_{(\varphi, \psi)}$ [see Eq. (3.2)] as the limit of the corresponding differences of the energy densities integrated over a finite volume as the volume invades the whole space. To prove this property we need some extra assumptions on the admissible $\varphi$. In this way (Corollary 2) we will be able to satisfy the important physical requirement that the energy scale does not have a physical meaning.

Theorem 6. Let the hypotheses of Theorem 5 be satisfied with the additional assumption

c) $\left|\nabla \varphi_{j}\right| \in L^{2}\left(\mathbb{R}^{s}\right), \quad j=1, \ldots, n$.

Then for all $x_{0} \in \mathbb{R}^{s}$ and for all $\omega \in C_{0}^{\infty}\left(\mathbb{R}^{s}\right)$ equal to 1 in a neighbourhood of the origin, one has

$$
E_{(\varphi, \psi)}\left(\varphi^{\prime}, \psi^{\prime}\right)=\lim _{R \rightarrow+\infty} \int_{\mathbb{R}^{s}}\left[K\left(\varphi^{\prime}, \psi^{\prime}\right)-K(\varphi, \psi)\right] \omega\left(\frac{x-x_{0}}{R}\right) d x .
$$

Proof. As in the proof of Theorem 5 it is immediate to check that

$$
\begin{aligned}
\int_{\mathbb{R}^{s}}\left[K\left(\varphi^{\prime}, \psi^{\prime}\right)-K(\varphi, \psi)\right] \omega\left(\frac{x-x_{0}}{R}\right) d x= & \int_{\mathbb{R}^{s}} V_{(\varphi, \psi)}(\chi, \zeta) \omega\left(\frac{x-x_{0}}{R}\right) d x \\
& -\left\langle h, \omega\left(\frac{x-x_{0}}{R}\right) \chi\right\rangle_{H^{-1}, H^{1}} \\
& -\left\langle\nabla \varphi,\left(\nabla \omega\left(\frac{x-x_{0}}{R}\right)\right) \chi\right\rangle_{L^{2}, L^{2}} \\
& \equiv I_{1}-I_{2}-I_{3} .
\end{aligned}
$$


Now $I_{1} \rightarrow \int_{\mathbb{R}^{s}} V_{(\varphi, \psi)}(\chi, \zeta) d x$, as $R \rightarrow+\infty$, by the dominated convergence theorem, $I_{2} \rightarrow\langle h, \chi\rangle_{H^{-1}, H^{1}}$ since $\omega\left(\frac{x-x_{0}}{R}\right) \chi \rightarrow \chi$, as $R \rightarrow+\infty$, in $Y_{1}, I_{3} \rightarrow 0$, as $R \rightarrow+\infty$, by assumption c). Corollary 2. Let the hypotheses of Theorem 6 be satisfied. Let $\left(\begin{array}{l}\alpha \\ \beta\end{array}\right) \in \mathscr{H}_{(\varphi, \psi)}$ and $\alpha$
be admissible with respect to $U$. Then

$$
E_{(\varphi, \psi)}\left(\varphi^{\prime}, \psi^{\prime}\right)=E_{(\alpha, \beta)}\left(\varphi^{\prime}, \psi^{\prime}\right)+E_{(\varphi, \psi)}(\alpha, \beta), \quad \forall\left(\begin{array}{l}
\varphi^{\prime} \\
\psi^{\prime}
\end{array}\right) \in \mathscr{H}_{(\varphi, \psi)} .
$$

Proof. We remark that $\alpha$ satisfies assumption c) of Theorem 6. Equality (3.13) is then an immediate consequence of Equation (3.11).

Corollary 2 has the obvious implication that the difference $E_{(\varphi, \psi)}\left(\varphi^{\prime}, \psi^{\prime}\right)-$ $E_{(\varphi, \psi)}\left(\varphi^{\prime \prime}, \psi^{\prime \prime}\right)$ does not depend on the choice of the element $\left(\begin{array}{l}\varphi \\ \psi\end{array}\right)$.

Besides the energy it is worth defining also the linear momentum.

Theorem 7. Let the hypotheses of Theorem 6 be satisfied, then the functional (linear momentum)

$$
\mathscr{H}_{(\varphi, \psi)} \ni\left(\begin{array}{c}
\varphi^{\prime} \\
\psi^{\prime}
\end{array}\right) \mapsto \boldsymbol{P}_{(\varphi, \psi)}\left(\varphi^{\prime}, \psi^{\prime}\right)=\int_{\mathbb{R}^{s}} \sum_{j=1}^{n}\left(\psi_{j}^{\prime} \nabla \varphi_{j}^{\prime}-\psi_{j} \nabla \varphi_{j}\right) d x
$$

is well defined and continuous on $\mathscr{H}_{(\varphi, \varphi)}$ (see Remark 4).

Proof. Obvious.

It is clear that, contrarily to the case of the total energy, now the quantity $\sum_{j=1}^{n} \psi_{j}^{\prime} \nabla \varphi_{j}^{\prime}$ belongs to $L^{1}\left(\mathbb{R}^{s}\right)$ without subtracting the momentum of the "background".

We will now give a brief description of the space-time translations.

Theorem 8. For all $(\tau, a) \in \mathbb{R} \times \mathbb{R}^{s}$ let us consider the map

$$
C^{(0)}(\mathbb{R} ; X) \ni\left(\begin{array}{c}
\varphi^{\prime}(t, x) \\
\psi^{\prime}(t, x)
\end{array}\right) \mapsto T_{\tau, a}\left(\begin{array}{c}
\varphi^{\prime} \\
\psi^{\prime}
\end{array}\right)=\left(\begin{array}{c}
\varphi^{\prime}(t+\tau, x+a) \\
\psi^{\prime}(t+\tau, x+a)
\end{array}\right) .
$$

Then

1) $T_{\tau, a}$ is continuous with values in $C^{(0)}(\mathbb{R} ; X)$.

2) If $U \in \mathscr{U}(X)$ and does not depend on the space variables, $T_{\tau, a}$ is a bijection of $\mathscr{F}_{U}$ (see the beginning of Section 2 ).

3) If $\left(\begin{array}{l}\varphi \\ \psi\end{array}\right) \in X$ satisfies the hypotheses of Theorem 6 with $U$ independent of the space variables, the map

$$
\left.\mathscr{H}_{(\varphi, \psi)} \ni\left(\begin{array}{c}
\varphi^{\prime} \\
\psi^{\prime}
\end{array}\right) \mapsto\left(T_{\tau, a}\left(\begin{array}{c}
\varphi^{\prime}(t) \\
\psi^{\prime}(t)
\end{array}\right)\right)\right|_{t=0}
$$


is a bijection of $\mathscr{H}_{(\varphi, \varphi)}\left[\right.$ here $\left(\begin{array}{l}\varphi^{\prime}(t) \\ \psi^{\prime}(t)\end{array}\right)$ is the solution of Equation $(1.2)$ with $\varphi^{\prime}(0)=\varphi^{\prime}$, $\left.\psi^{\prime}(0)=\psi^{\prime}\right]$.

Proof. Assertion 1) is trivial. Assertion 2) follows, by the uniqueness Theorem 1, from the equation

$$
\begin{aligned}
\left(\begin{array}{l}
\varphi^{\prime}(t+\tau, x+a) \\
\psi^{\prime}(t+\tau, x+a)
\end{array}\right)= & W(t)\left[W(\tau)\left(\begin{array}{l}
\varphi^{\prime}(0, x+a) \\
\psi^{\prime}(0, x+a)
\end{array}\right)\right. \\
& \left.+\int_{0}^{t+\tau} W(t-s)\left(\begin{array}{c}
0 \\
-\nabla_{z} U\left(\varphi^{\prime}(s, x+a)\right)
\end{array}\right) d s\right] \\
= & W(t)\left(\begin{array}{c}
\varphi^{\prime}(\tau, x+a) \\
\psi^{\prime}(\tau, x+a)
\end{array}\right)+\int_{0}^{t} W(t-\sigma)\left(\begin{array}{c}
0 \\
-\nabla_{z} U\left(\varphi^{\prime}(\tau+\sigma, x+a)\right.
\end{array}\right) d \sigma
\end{aligned}
$$

which is a consequence of Equation (1.2) and of the fact that $W(t)$ is a convolution operator. Assertion 3) follows easily from the identities

$$
\begin{aligned}
& \psi^{\prime}(\tau, x+a)-\psi^{\prime}(x)=\left(\psi^{\prime}(\tau, x+a)-\psi^{\prime}(x+a)\right)+\left(\psi^{\prime}(x+a)-\psi^{\prime}(x)\right) \\
& \varphi^{\prime}(\tau, x+a)-\varphi^{\prime}(x)=\left(\varphi^{\prime}(\tau, x+a)-\varphi^{\prime}(x+a)\right)+\left(\varphi^{\prime}(x+a)-\varphi^{\prime}(x)\right) .
\end{aligned}
$$

In fact, $\psi^{\prime}(x+a)-\psi^{\prime}(x)$ and $\nabla_{x}\left(\varphi^{\prime}(x+a)-\varphi^{\prime}(x)\right)$ obviously belong to $Y_{0}$. Moreover, $\psi^{\prime}(\tau, x+a)-\psi^{\prime}(x+a)$ and $\varphi^{\prime}(\tau, x+a)-\varphi^{\prime}(x+a)$ belongs to $Y_{0}$ and to $Y_{1}$, respectively, by the definition of $\mathscr{H}_{(\varphi, \psi)}$. Finally $\left(\varphi^{\prime}(x+a)-\varphi^{\prime}(x)\right) \in Y_{0}$ as a byproduct of the mean value theorem and of $\left|\nabla \varphi_{j}\right| \in L^{2}\left(\mathbb{R}^{s}\right), j=1, \ldots, n$.

It may have some interest to compare the energy of pairs of elements belonging to different Hilbert sectors because their relative energy may be finite, as e.g. in the case of degeneracy

Definition 6. Let $\mathscr{H}_{(\varphi, \psi)}$ and $\mathscr{H}_{(\alpha, \beta)}$ be two different Hilbert sectors with $\left(\begin{array}{l}\varphi \\ \psi\end{array}\right),\left(\begin{array}{l}\alpha \\ \beta\end{array}\right)$ satisfying the hypotheses of Theorem 6 . We say that these sectors have relatively finite energy if for all $x_{0} \in \mathbb{R}^{s}$ and for all $\omega \in C_{0}^{\infty}\left(\mathbb{R}^{s}\right)$ equal to 1 in a neighbourhood of the origin, the limit

$$
\lim _{R \rightarrow+\infty} \int_{\mathbb{R}^{s}}\left[K\left(\varphi^{\prime}, \psi^{\prime}\right)-K\left(\alpha^{\prime}, \beta^{\prime}\right)\right] \omega\left(\frac{x-x_{0}}{R}\right) d x
$$

exists, finite, independent of $x_{0}$ and $\omega$, for all

$$
\left(\begin{array}{c}
\varphi^{\prime} \\
\psi^{\prime}
\end{array}\right) \in \mathscr{H}_{(\varphi, \psi)}, \quad\left(\begin{array}{c}
\alpha^{\prime} \\
\beta^{\prime}
\end{array}\right) \in \mathscr{H}_{(\alpha, \beta)}
$$

To establish that the sectors $\mathscr{H}_{(\varphi, \psi)}$ and $\mathscr{H}_{(\alpha, \beta)}$ have relatively finite energy it will be enough, as a consequence of Theorem 6 and relation (3.13), to check the existence and the properties of the limit (3.19) only for one pair of elements. 
In particular it will be sufficient to verify that the limit

$$
\lim _{R \rightarrow \infty} \int_{\mathbb{R}^{s}}[U(x, \varphi(x))-U(x, \alpha(x))] \omega\left(\frac{x-x_{0}}{R}\right) d x
$$

exists, finite, independent of $x_{0}$ and $\omega$.

The results of this Section allow a rigorous treatment of the Hilbert sectors based on constant fields. Precisely we have the following

Theorem 9. Let $U \in \mathscr{U}(X)$, independent of the space variables and such that all constant functions $\varphi=c$ are admissible with respect to $U$. Then

1) $\left(\begin{array}{l}c \\ 0\end{array}\right)$ belongs to some Hilbert sector iff $\nabla_{z} U(c)=0$ (Goldstone's theorem) and, in this case, the energy functional takes the form

$$
E_{(c, 0)}\left(\varphi^{\prime}, \psi^{\prime}\right)=\int_{\mathbb{R}^{s}}\left[\sum_{j=1}^{n} \frac{1}{2}\left(\left|\nabla \varphi_{j}^{\prime}\right|^{2}+\psi_{j}^{\prime 2}\right)+U\left(\varphi^{\prime}\right)-U(c)\right] d x
$$

2) The Hilbert sectors $\mathscr{H}_{(c, 0)}, \mathscr{H}_{(\bar{c}, 0)}$ have relatively finite energy iff $U(c)=U(\bar{c})$. Proof. The first part of assertion 1) is a consequence of Theorems 3, 4, while Equation (3.21) follows from Theorem 6. Assertion 2) is a consequence of Definition 6 and of Equation (3.20).

A generalization of the above situation $(\varphi=c)$ is provided by the case in which $\varphi$ satisfies the equations $\Delta \varphi-\nabla_{z} U(x, \varphi)=0$ (soliton-like solutions). Some results in this direction, for $s=1$, are collected in Appendix $C$.

\section{Dynamical Charges}

The structure of Hilbert space sectors allows us to introduce as an invariant of each sector the "behaviour at $\infty$ " of its elements. In this Section we will show that for all $\left(\begin{array}{l}\varphi^{\prime} \\ \psi^{\prime}\end{array}\right) \in \mathscr{H}_{(\varphi, \psi)}$ the difference $\varphi^{\prime}-\varphi$ tends to zero at $\infty$ in almost all directions, and therefore if $\varphi$ has a limit at $\infty$ in almost all directions, $\varphi^{\prime}$ has the same limit. This will enable us to define a dynamical charge associated to $\mathscr{H}_{(\varphi, \psi)}$ which is preserved in time. The idea of associating a charge to the asymptotic behaviour of solutions of non-linear evolution equations has been first discussed by Finkelstein and Misner [4]. The framework constructed in the previous permits a rigorous treatment of this concept.

Lemma 5. Let $\varphi \in H^{1}\left(\mathbb{R}^{s}\right)$ and let us define

$$
\tilde{\varphi}(r, \omega) \equiv \varphi(x), \quad x=r \omega, \quad r>0, \quad \omega \in \mathbb{S}^{s-1} .
$$

Then for almost all $\omega \in \mathbb{S}^{s-1}$ (the unit sphere in $\mathbb{R}^{s}$ ) the function $r \mapsto \varphi^{2}(r, \omega)$ is continuous and satisfies the estimate

$$
r^{(s-1) / 2}\left|\varphi^{\sim}(r, \omega)\right|=o(1), \quad r \rightarrow+\infty .
$$


Proof. By Fubini-Tonelli's Theorem there is a subset $T_{1} \subset \mathbb{S}^{s-1}$ of zero measure in $\mathbb{S}^{-1}$ such that

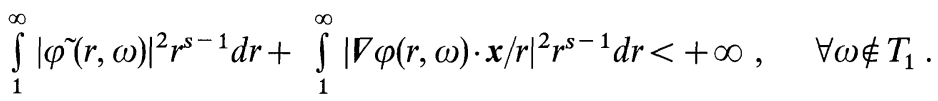

We can always find a sequence $\varphi_{j} \in C_{0}^{\infty}\left(\mathbb{R}^{s}\right)$ such that $\varphi_{j} \rightarrow \varphi$ in $H^{1}\left(\mathbb{R}^{s}\right)$ and for which

$$
\begin{aligned}
& \lim _{j \rightarrow \infty} \int_{1}^{\infty}\left|\tilde{\varphi_{j}}(r, \omega)-\tilde{\varphi^{2}}(r, \omega)\right|^{2} r^{s-1} d r \\
& +\int_{1}^{\infty}\left|\left(\nabla \varphi_{j}(r, \omega)-\nabla \varphi(r, \omega)\right) \cdot \boldsymbol{x} / r\right|^{2} r^{s-1} d r=0
\end{aligned}
$$

for all $\omega \in \mathbb{S}^{s-1} \backslash T_{2}$, where $T_{2}$ is a suitable set of zero measure independent of $j[10]$. From now on we fix $\omega \notin T_{1} \cup T_{2}$. There is a sub-sequence of $\left\{\varphi_{j}\right\}$ (depending on $\omega)$, still denoted by $\left\{\varphi_{j}\right\}$, such that

$$
\tilde{\varphi_{j}}(r, \omega) \rightarrow \tilde{\varphi}(r, \omega) \text { a.e. in }[1, \infty[\text { as } j \rightarrow \infty .
$$

Therefore

$$
\tilde{\varphi(r, \omega)}=\tilde{\varphi\left(r_{0}, \omega\right)}+\int_{r_{0}}^{r} \boldsymbol{\nabla} \varphi \cdot \boldsymbol{x} / \sigma d \sigma \quad \text { a.e. }
$$

as a consequence of the corresponding equations for the $\tilde{\varphi_{j}}$ 's. This establishes the continuity of $\tilde{\varphi}(r, \omega)$ conveniently redefined on a set of zero measure in $r$.

On the other hand, the estimate

$$
\begin{aligned}
\left|\left[\left|\varphi_{j}\right|^{2} r^{s-1}\right]_{r=r_{0}}^{r=r}\right|= & \left|\int_{r_{0}}^{r} \frac{d}{d \sigma}\left(\left|\varphi_{j}(\sigma \omega)\right|^{2} \sigma^{s-1}\right) d \sigma\right| \\
\leqq & 2\left(\int_{r_{0}}^{r}\left|\varphi_{j}\right|^{2} \sigma^{s-1} d \sigma\right)^{1 / 2}\left(\int_{r_{0}}^{r}\left|d \varphi_{j} / d \sigma\right|^{2} \sigma^{s-1} d \sigma\right)^{1 / 2} \\
& +\frac{s-1}{r_{0}} \int_{r_{0}}^{r}\left|\varphi_{j}\right|^{2} \sigma^{s-1} d \sigma
\end{aligned}
$$

yields, in the limit $j \rightarrow \infty$

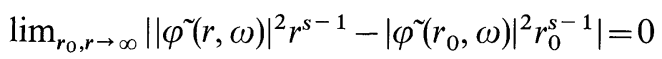

which implies Equation (4.2), by Equation (4.3).

Definition 7. Let $\mathscr{H}_{(\varphi, \psi)}$ be a Hilbert space sector (see Definition 5). If there is a map $a: \mathbb{S}^{s-1} \rightarrow \mathbb{R}^{n}$ such thàt $\tilde{\varphi}(r, \omega) \equiv \varphi(r \omega) \rightarrow a(\omega)$ as $r \rightarrow+\infty$, almost everywhere in $\mathbb{S}^{s-1}$, we will say that a is the charge of $\mathscr{H}_{(\varphi, \varphi)}$.

The above Definition makes sense by Lemma 5 since $\mathscr{H}_{(\varphi, \psi)} \subset\left(\begin{array}{l}\varphi \\ \psi\end{array}\right)+Y$. Moreover, by definition of sector, for all $\left(\begin{array}{c}\varphi^{\prime} \\ \psi^{\prime}\end{array}\right) \in \mathscr{H}_{(\varphi, \psi)}, \varphi^{\sim \prime}(t ; r, \omega) \equiv \varphi^{\prime}(t, r \omega) \rightarrow a(\omega)$ a.e. in $\mathbb{S}^{s-1}$ as $r \rightarrow \infty, \forall t \in \mathbb{R}$ [where $\varphi^{\prime}(t, x)$ is the solution of Equation (1.2) with 
$\left.\varphi^{\prime}(0, x)=\varphi^{\prime}, \psi^{\prime}(0, x)=\psi^{\prime}\right]$. This fact may be interpreted as the conservation of charge. It is worthwhile to remark that the existence of non-trivial charges relies crucially on the existence of non-trivial Hilbert sectors (see Section 2, especially Theorem 4).

For $s \geqq 3$ the existence of the charge is guaranteed for those sectors $\mathscr{H}_{(\varphi, \psi)}$ for which $\nabla \varphi_{j} \in L^{2}\left(\mathbb{R}^{s}\right), j=1, \ldots n$, as a consequence of the following

Lemma 6. Let $s \geqq 3, \varphi \in H_{\mathrm{loc}}^{1}\left(\mathbb{R}^{s}\right)$ with $\partial \varphi / \partial x_{j} \in L^{2}\left(\mathbb{R}^{s}\right), j=1, \ldots s$, and define

$$
\tilde{\varphi}(r, \omega) \equiv \varphi(x), \quad x=r \omega, \quad r>0, \quad \omega \in \mathbb{S}^{s-1} .
$$

Then for almost all $\omega \in \mathbb{S}^{s-1}$ (the unit sphere of $\mathbb{R}^{s}$ ) the function $r \mapsto \varphi(r, \omega)$ is continuous, has a finite limit $\tilde{\varphi}(\infty, \omega)$ as $r \rightarrow+\infty$ and

$$
r^{(s-2) / 2}|\tilde{\varphi}(r, \omega)-\tilde{\varphi}(\infty, \omega)|=o(1), \quad \text { as } \quad r \rightarrow+\infty .
$$

Proof. It is possible, by using a mollifier technique, to find a sequence $\left\{\varphi_{j}\right\}, \varphi_{j} \in C^{\infty}\left(\mathbb{R}^{s}\right)$, with $\partial \varphi_{j} / \partial x_{k} \in L^{2}\left(\mathbb{R}^{s}\right), \forall j, k$, such that $\varphi_{j} \rightarrow \varphi$ in $L_{\mathrm{loc}}^{2}\left(\mathbb{R}^{s}\right)$ and $\partial \varphi_{j} / \partial x_{k} \rightarrow \partial \varphi / \partial x_{k}$ in $L^{2}\left(\mathbb{R}^{s}\right), \forall k$. Now, by the same kind of arguments used in the

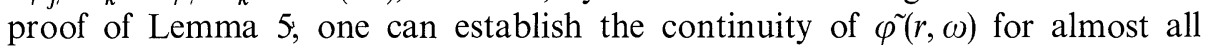
$\omega \in \mathbb{S}^{s-1}$. On the other hand, the estimate $\left(r>r_{0} \geqq 1\right)$

$$
\begin{aligned}
\left|\tilde{\varphi}_{j}(r, \omega)-\tilde{\varphi_{j}}\left(r_{0}, \omega\right)\right| & \leqq \int_{r_{0}}^{r}\left|d \varphi_{j}(\sigma, \omega) / d \sigma\right| d \sigma \\
& \leqq\left(\int_{r_{0}}^{r}\left|d \varphi_{j}(\sigma, \omega) / d \sigma\right|^{2} \sigma^{s-1} d \sigma\right)^{1 / 2}\left(\int_{r_{0}}^{r} \sigma^{1-s} d \sigma\right)^{1 / 2}
\end{aligned}
$$

yields, in the limit $j \rightarrow \infty$,

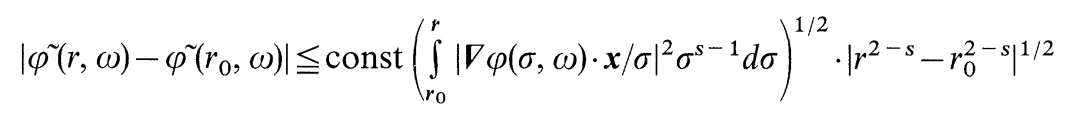

from which Equation (4.8) follows.

The results presented in Appendix $\mathrm{C}$ provide sufficient conditions for the existence of the charge $a$ in the case $s=1$.

\section{Internal Symmetries and Spontaneous Symmetry Breaking}

In this Section we will discuss the concept of internal symmetry and we will show how, within our framework, the mechanism of symmetry breaking can be understood in a rigorous way. It is convenient to start with the following natural definition of internal symmetry

Definition 8. Let $g: \mathbb{R}^{n} \rightarrow \mathbb{R}^{n}$ be a diffeomorphism of class $C^{(2)}$ such that the map

$$
T_{g}:\left(\begin{array}{l}
\varphi(x) \\
\psi(x)
\end{array}\right) \mapsto\left(\begin{array}{c}
g(\varphi(x)) \\
J_{g}(\varphi(x)) \psi(x)
\end{array}\right)
$$


where $J_{g}$ is the Jacobian matrix of $g$, is continuous from $X$ to $X$. We will say that $T_{g}$ is a local internal symmetry of Equation (1.2) if, for every solution $\left(\begin{array}{l}\varphi(t) \\ \psi(t)\end{array}\right) \in C^{(0)}$ $(\mathbb{R} ; X)$ of Equation (1.2), $T_{g}\left(\begin{array}{l}\varphi(t) \\ \psi(t)\end{array}\right)$ is again a solution of Equation (1.2).

The next two theorems give a simple characterization of internal symmetries under mild assumptions on the interaction.

Theorem 10. Let $U \in \mathscr{U}(X)$ with the properties

i) $U(x, 0)=0 \forall x \in \mathbb{R}^{s}, U(x, z) \neq 0$.

ii) For any $C^{\infty}\left(\mathbb{R}^{s}\right)$-initial data $\left(\begin{array}{l}\varphi(x) \\ \psi(x)\end{array}\right)$ the corresponding continuous solution of Equation (1.2) $\left(\begin{array}{l}\varphi(t, x) \\ \psi(t, x)\end{array}\right)$ is such that $\varphi_{j}(t, x) \in C^{(2)}\left(\mathbb{R} \times \mathbb{R}^{s}\right), j=1, \ldots n$.

Then, if $T_{g}$ is a local internal symmetry, the map $g$ is an affine transformation with $J_{g}^{T} J_{g}=\lambda_{g} \mathbb{1}_{\mathbb{R}^{n}}$ for some constant $\lambda_{g}$, and for all spheres $\Omega$

$$
\int_{\Omega}\left[K\left(g \circ \varphi, J_{g} \psi\right)-\lambda_{g} K(\varphi, \psi)-U(x, g(0))\right] d x=0
$$

for all $\left(\begin{array}{l}\varphi \\ \psi\end{array}\right) \in X[$ for the definition of $K$ see Equation (3.1)].

Proof. If $\left(\begin{array}{l}\varphi(t, x) \\ \psi(t, x)\end{array}\right)$ is the solution of Equation (1.2) with $C^{\infty}$-initial data $\left(\begin{array}{l}\varphi(x) \\ \psi(x)\end{array}\right)$. then condition ii) and Definition 8 imply

$$
\begin{aligned}
& \square \varphi_{j}(t, x)+\frac{\partial U}{\partial z_{j}}(x, \varphi(t, x))=0 \\
& \square g(\varphi(t, x))+\frac{\partial U}{\partial z_{j}}(x, g(\varphi(t, x))=0 .
\end{aligned}
$$

A combination of the two Equations (5.3) yields

$$
\begin{aligned}
& \sum_{j, k=1}^{n} \frac{\partial^{2} g_{l}}{\partial z_{j} \partial z_{k}}(\varphi) \partial^{\mu} \varphi_{j} \partial_{\mu} \varphi_{k}-\sum_{j=1}^{n} \frac{\partial g_{l}}{\partial z_{j}}(\varphi) \frac{\partial U}{\partial z_{j}}(x, \varphi)+\frac{\partial U}{\partial z_{l}}(x, g(\varphi))=0, \\
& l=1, \ldots n
\end{aligned}
$$

Now, by Definition 8, Equation (5.4) has to hold for all times and for all initial data. A suitable choice of such data in Equation (5.4) shows that

$$
\frac{\partial^{2} g_{l}}{\partial z_{j} \partial z_{k}}(c)=0, \quad l=1, \ldots n, \quad c \in \mathbb{R}^{n},
$$

i.e. $g$ is of the form

$$
g(z)=A z+a
$$

with $A$ an invertible matrix and $a \in \mathbb{R}^{n}$. 
Then, from Equation (5.4) it follows

$$
\nabla_{z}\left[U(x, A z+a) \mathbb{1}_{\mathbb{R}^{n}}-U(x, z) A^{T} A\right]=0
$$

and therefore

$$
U(x, A z+a) \mathbb{1}_{\mathbb{R}^{n}}=U(x, z) A^{T} A+U(x, a) \mathbb{1}_{\mathbb{R}^{n}} .
$$

Since $U$ is non-trivial we conclude that $A^{T} A$ is a multiple of the identity. At this point the validity of Equation (5.2) can be immediately verified.

Remark 5. It is easy to see that the set of local internal symmetries $\left\{T_{g}\right\}$ is a group and that $T_{g} \rightarrow \lambda_{g}$ is a one-dimensional representation of such a group.

Remark 6. It can be checked directly on Equation (1.2) that any map $g$ of the form (5.6) and satisfying Equation (5.8) defines a local internal symmetry.

Remark 7. Conditions on the potential $U$ ensuring the validity of hypothesis ii) of Theorem 10 have been discussed in [6]. Hypothesis i) can always be satisfied by an inessential redefinition of $U$. Finally, in the case $U \equiv 0$ the only internal symmetries are the affine transformations.

Theorem 11. Let $g: \mathbb{R}^{n} \rightarrow \mathbb{R}^{n}$ be a diffeomorphism of class $C^{(2)}$ such that Equation (5.1) defines a map from $X$ to $X$. Then, if there exists a real constant $\lambda$ and measurable function $f: \mathbb{R}^{s} \rightarrow \mathbb{R}$ such that

$$
\int_{\Omega}\left[K\left(g \circ \varphi, J_{g}(\varphi) \psi\right)-\lambda K(\varphi, \psi)-f(x)\right] d x=0
$$

for all spheres $\Omega$ and for all $\left(\begin{array}{c}\varphi \\ \psi\end{array}\right) \in X$, the mapping $T_{g}$ is a local internal symmetry. Proof. From Equation (5.2)' it follows that $f(x)=U(x, g(0))$ and that

$$
\begin{aligned}
& \frac{1}{2} \sum_{k=1}^{n}\left(\left|\nabla\left(g_{k}(\varphi(x))\right)\right|^{2}-\lambda\left|\nabla \varphi_{k}(x)\right|^{2}\right)+U(x,(g \circ \varphi)(x))-\lambda U(x, \varphi(x))-f(x) \\
& =-\frac{1}{2}\left(\left|J_{g}(\varphi(x)) \psi(x)\right|^{2}-\lambda|\psi(x)|^{2}\right) .
\end{aligned}
$$

By varying $\varphi$ and $\psi$ independently, Equation (5.9) implies

$$
J_{g}^{T}(z) J_{g}(z)=\lambda \mathbb{1}_{\mathbb{R}^{n}}, \quad \forall z \in \mathbb{R}^{n}
$$

and

$$
U(x, g(z))=\lambda U(x, z)+U(x, g(0)) .
$$

The map $g_{1}(z) \equiv \lambda^{-1 / 2} g(z)$ has the property that $J_{g_{1}}^{T}(z) J_{g_{1}}(z)=\mathbb{1}_{\mathbb{R}^{n}}, \forall z$ and therefore, by the mean value theorem,

$$
\left|g_{1}\left(z^{\prime}\right)-g_{1}\left(z^{\prime \prime}\right)\right|=\left|z^{\prime}-z^{\prime \prime}\right|, \quad \forall z^{\prime}, z^{\prime \prime} \in \mathbb{R}^{n} .
$$

By a well known result Equation (5.14) implies that $g_{1}$ is an affine transformation. By Remark 5, $g$ defines a local internal symmetry. 
Definition 9. A local internal symmetry $T_{g}$ is called a global internal symmetry of the Hilbert space sector $\mathscr{H}_{(\varphi, \psi)}$ if

$$
T_{g}\left(\begin{array}{c}
\varphi^{\prime} \\
\psi^{\prime}
\end{array}\right) \in \mathscr{H}_{(\varphi, \psi)}, \quad \forall\left(\begin{array}{c}
\varphi^{\prime} \\
\psi^{\prime}
\end{array}\right) \in \mathscr{H}_{(\varphi, \psi)}
$$

A local internal symmetry is said to be spontaneously broken in the Hilbert sector $\mathscr{H}_{(\varphi, \psi)}$ if there exists a $\left(\begin{array}{c}\varphi^{\prime} \\ \psi^{\prime}\end{array}\right) \in \mathscr{H}_{(\varphi, \psi)}$ such that $T_{g}\left(\begin{array}{c}\varphi^{\prime} \\ \psi^{\prime}\end{array}\right) \notin \mathscr{H}_{(\varphi, \psi)}$.

The next Theorem shows that to have a global symmetry it is enough to check condition (5.13) on a single element of the sector. Consequently, if $T_{g}$ is spontaneously broken, then $T_{g}\left(\begin{array}{l}\varphi^{\prime} \\ \psi^{\prime}\end{array}\right) \notin \mathscr{H}_{(\varphi, \psi)}$ for all elements $\left(\begin{array}{c}\varphi^{\prime} \\ \psi^{\prime}\end{array}\right)$ belonging to $\mathscr{H}_{(\varphi, \psi)}$.

Theorem 12. Let $T_{g}$ be a local internal symmetry induced by an affine transformation and $\mathscr{H}_{(\varphi, \psi)}$ a Hilbert sector. If for some $\left(\begin{array}{c}\varphi^{\prime} \\ \psi^{\prime}\end{array}\right) \in \mathscr{H}_{(\varphi, \psi)} T_{g}\left(\begin{array}{c}\varphi^{\prime} \\ \psi^{\prime}\end{array}\right) \in \mathscr{H}_{(\varphi, \psi)}$, then $T_{g}$ is a global internal symmetry (of $\left.\mathscr{H}_{(\varphi, \varphi)}\right)$.

Proof. The proof is an immediate consequence of the structure of $g$ and of the definition of Hilbert sector.

Now any Hilbert space sector of the form $\mathscr{H}_{(\varphi, \psi)}=\left(\begin{array}{l}\varphi \\ \psi\end{array}\right)+Y$ carries an obvious Hilbert structure (see Remark 4), i.e. that induced by the identification $i: \mathscr{H}_{(\varphi, \varphi)} \rightarrow Y$, $i\left(\begin{array}{l}\varphi^{\prime} \\ \psi^{\prime}\end{array}\right)=\left(\begin{array}{l}\varphi^{\prime}-\varphi \\ \psi^{\prime}-\psi\end{array}\right)$. Therefore, if $T_{g}$ is a global internal symmetry for $\mathscr{H}_{(\varphi, \psi)}$, with $g(z)=A z+a$ and $A^{T} A=\lambda_{g} \mathbb{1}_{\mathbb{R}^{n}}$, then the map $U_{g}: \mathscr{H}_{(\varphi, \varphi)} \rightarrow \mathscr{H}_{(\varphi, \varphi)}, U_{g}\left(\begin{array}{c}\varphi^{\prime} \\ \psi^{\prime}\end{array}\right)=\left(\begin{array}{c}\varphi \\ \psi\end{array}\right)+$ $\lambda_{g}^{-1 / 2}\left(\begin{array}{c}A\left(\varphi^{\prime}-\varphi\right) \\ A\left(\psi^{\prime}-\psi\right)\end{array}\right)$ is unitary.

\section{Appendix A}

In this Appendix we state and prove an existence and uniqueness theorem for the solution of an abstract integral equation of the type

$$
u(t)=W(t) u_{0}+L(t)+\int_{0}^{t} W(t-s) f(s, u(s)) d s
$$

under suitable assumptions on $W(t), L(t)$, and $f(t, v)$. The proof, which is obtained by a straightforward modification of Segal's approach [7], is reproduced here for the convenience of the reader 
Theorem A. Let $B$ be a Banach space with norm $\|\cdot\|$. Suppose we are given

i) A strongly continuous one-parameter group $(W(t))_{t \in \mathbb{R}}($ with $W(0)=I)$ of linear bounded operators on $B$.

ii) $A$ map $f \in C^{0)}(\mathbb{R} \times B ; B)$ such that, for any $T, \varrho>0$, there exists a positive constant $C(T, \varrho)$ for which the inequality

$$
\sup _{0 \leqq|t| \leqq T}\|f(t, u)-f(t, v)\| \leqq C(T, \varrho)\|u-v\|
$$

holds for all $u, v \in B$ with $\|u\| \leqq \varrho,\|v\| \leqq \varrho$.

iii) $A$ continuous map

$$
L: \mathbb{R} \rightarrow B
$$

such that $L(0)=0$.

Furthermore we suppose that for any $T>0$ if $u \in C^{0)}([0, T[; B)$ (resp. $\left.\left.u \in C^{0)}(]-T, 0\right] ; B\right)$ is a solution of the integral Equation (A.1) on the interval $[0, T[$ (resp. $]-T, 0]$ ) with $u_{0} \in B$, then there exists a sequence $0<t_{j} \uparrow T$ (resp. $\left.t_{j} \downarrow-T\right) \mathrm{s} \mu \mathrm{ch}$ that

$$
\sup _{t_{j}}\left\|u\left(t_{j}\right)\right\|<\infty \text {. }
$$

Under these assumptions the integral Equation (A.1) has a unique solution $u \in C^{(0)}(\mathbb{R} ; B)$ for any initial data $u_{0} \in B$.

Proof. For obvious reasons it is enough to prove that Equation (A.1) has a unique solution $u \in C^{0)}\left(\left[0,+\infty[; B)\right.\right.$ for any initial data $u_{0} \in B$. It is useful to recall that as a consequence of hypothesis i), [11], there exist constants $A \geqq 1$ and $\omega>0$ such that

$$
\|W(t) u\| \leqq A e^{\omega|t|}\|u\|, t \in \mathbb{R}, u \in B .
$$

Uniqueness is now obvious because if $u_{1}, u_{2} \in C^{(0)}([0, T] ; B), 0<T<\infty$, are solutions of Equation (A.1) with the same initial data, then

$$
\left\|u_{1}(t)-u_{2}(t)\right\| \leqq A e^{\omega T} C(T, \varrho) \int_{0}^{T}\left\|u_{1}(s)-u_{2}(s)\right\| d s, t \in[0, T],
$$

where $\varrho=\sup _{0 \leqq t \leqq T, k=1,2}\left\|u_{k}(t)\right\|$, and therefore Gronwall's lemma implies $u_{1}(t)=u_{2}(t), t \in[0, \bar{T}]$.

To establish existence we first proceed to the construction of a perturbative solution. Given $T, \varrho>0$ we define the space

$$
E(T, \varrho)=\left\{\left.u \in C^{(0)}([0, T] ; B)\left|\sup _{0 \leqq t \leqq T}\|u(t)\| \equiv\right| u\right|_{T} \leqq \varrho\right\} .
$$

Equipped with the distance $d(u, v) \equiv|u-v|_{T}, E(T, \varrho)$ is a complete metric space. We now show that for any $u_{0} \in B,\left\|u_{0}\right\| \leqq \varrho / 2 A$, there exists a $\bar{T}>0$ (depending only on $\varrho$ ) such that Equation (A.1) has a (unique) solution $u \in E(\bar{T}, \varrho)$. Consider the operator

$$
(S u)(t)=W(t) u_{0}+L(t)+\int_{0}^{t} W(t-s) f(s, u(s)) d s, u \in E(T, \varrho) .
$$


The estimates

$$
\begin{aligned}
& \|(S u)(t)\| \leqq A e^{\omega t}\left\|u_{0}\right\|+\|L(t)\|+A e^{\omega t} t\left[\sup _{0 \leqq t \leqq T}\|f(s, 0)\|+C(T, \varrho) \varrho\right] \\
& \left\|\left(S u_{1}\right)(t)-\left(S u_{2}\right)(t)\right\| \leqq A e^{\omega t} C(T, \varrho) \int_{0}^{t}\left\|u_{1}(s)-u_{2}(s)\right\| d s
\end{aligned}
$$

imply the existence of a $0<\bar{T} \leqq T$ such that the operator $S$ is a contraction on $E(\bar{T}, \varrho)$. By Banach's theorem on contractions $S$ has a unique fixed point which is a solution of Equation (A.1).

To complete the proof of the Theorem we are going to show that any solution $u \in C^{(0)}([0, T[; B), 0<T<\infty$, of Equation (A.1) can be continued beyond $T$. For this purpose let us consider the integral equation

$$
v(t)=W(t-\bar{t}) u(\bar{t})+L(t)-W(t-\bar{t}) L(\bar{t})+\int_{\bar{t}}^{t} W(t-s) f(s, u(s)) d s
$$

where $t \in\left[\bar{t},+\infty\left[\right.\right.$ and $\bar{t}$ can be any of the $t_{j}$ 's of Inequality (A.3). If we can show that Equation (A.9) has a continuous solution $v$ on an interval $[\bar{t}, \bar{t}+\tau]$ with $\tau$ independent of $\bar{t}$, then the function

$$
\varphi(t)=\left\{\begin{array}{ll}
u(t), & 0 \leqq t \leqq \bar{t} \\
v(t), & \bar{t} \leqq t \leqq \bar{t}+\tau
\end{array} \quad \bar{t}+\tau>T\right.
$$

continues the solution beyond $T$. This follows from the equality

$$
\begin{aligned}
& \varphi(t)-\left(W(t) u_{0}+\int_{0}^{t} W(t-s) f(s, \varphi(s)) d s\right) \\
& =W(t-\bar{t})\left\{u(\bar{t})-L(\bar{t})-\int_{0}^{\bar{t}} W(\bar{t}-s) f(s, u(s)) d s-W(\bar{t}) u_{0}\right\}=0 .
\end{aligned}
$$

The existence of a perturbative solution of Equation (A.9) proceeds exactly as above. The crucial fact, that we can choose $\tau$ independent of the $t_{j}$ 's, is a consequence of the estimate

$$
\begin{aligned}
& \|W(t-\bar{t}) u(\bar{t})+L(t)-W(t-\bar{t}) L(\bar{t})\| \\
& \leqq A e^{\omega(t-\bar{t})} \sup _{j}\left\|u\left(t_{j}\right)\right\|+\|L(t)+W(t-\bar{t}) L(\bar{t})\|
\end{aligned}
$$

and of the uniform continuity of the function $(\theta, \sigma) \rightarrow L(\theta+\sigma)-W(\theta) L(\sigma)$ on the compact subsets of $\mathbb{R}^{+} \times \mathbb{R}^{+}$.

\section{Appendix B}

To recognize that $\chi(t)$ is $L^{2}$-differentiable we first integrate Equation (2.17)

$$
\left(\begin{array}{l}
P_{r} \chi(t) \\
P_{r} \zeta(t)
\end{array}\right)=\left(\begin{array}{c}
P_{r} \chi_{0} \\
P_{r} \zeta_{0}
\end{array}\right)+\int_{0}^{t}\left(\begin{array}{l}
P_{r} \zeta(s)+P_{r} \psi_{0} \\
\Delta P_{r} \chi(s)+P_{r} h-P_{r} \nabla_{z} F_{\varphi_{0}}(x, \chi(s))
\end{array}\right) d s
$$


and then apply the linear bounded operator $\left(\begin{array}{ll}0 & 0 \\ 1 & 0\end{array}\right) \otimes \mathbb{1}_{\mathbb{C}^{n}}$ to (B.1)

$$
\left(\begin{array}{c}
0 \\
P_{r} \chi(t)
\end{array}\right)=\left(\begin{array}{c}
0 \\
P_{r} \chi_{0}
\end{array}\right)+\int_{0}^{t}\left(\begin{array}{c}
0 \\
P_{r} \zeta(s)+P_{r} \psi_{0}
\end{array}\right) d s .
$$

It is now clear that, letting $r \rightarrow \infty, d \chi(t) / d t=\zeta(t)+\psi_{0}$.

We now show that for any $\varrho \in Y_{1}, F_{\varphi_{0}}(x, \varrho(x)) \in L^{1}\left(\mathbb{R}^{s}\right)$. Successive applications of the mean value theorem and Hölder inequality yield

$$
\int_{\mathbb{R}^{s}}\left|F_{\varphi_{0}}(x, \varrho(x))\right| d x \leqq \sup _{0 \leqq \sigma \leqq 1}\left\|\nabla_{z} F_{\varphi_{0}}(x, \sigma \varrho(x)) ; Y_{0}\right\| \cdot\left\|\varrho ; Y_{0}\right\|
$$

which is obviously finite because $F_{\varphi_{0}} \in \mathscr{U}(Y)$.

Finally we prove that the function

$$
C(t)=\int_{\mathbb{R}^{s}} F_{\varphi_{0}}(x, \chi(t, x)) d x
$$

is differentiable with

$$
d C(t) / d t=\left\langle\zeta(t)+\psi_{0}, \nabla_{z} F_{\varphi_{0}}(x, \chi(t))\right\rangle .
$$

It is convenient to start from the identity

$$
\begin{aligned}
& \frac{C(t+\Delta t)-C(t)}{\Delta t}=\int_{\mathbb{R}^{s}}\left\{\frac{F_{\varphi_{0}}(x, \chi(t+\Delta t, x))-F_{\varphi_{0}}(x, \chi(t, x))}{\Delta t}\right. \\
& \left.-\sum_{j=1}^{n} \frac{\partial F_{\varphi_{0}}}{\partial z_{j}}(x, \chi(t, x)) \frac{\chi_{j}(t+\Delta t, x)-\chi_{j}(t, x)}{\Delta t}\right\} d x \\
& +\int_{\mathbb{R}^{s}} \sum_{j=1}^{n} \frac{\partial F_{\varphi_{0}}}{\partial z_{j}}(x, \chi(t, x))\left[\frac{\chi_{j}(t+\Delta t, x)-\chi_{j}(t, x)}{\Delta t}-\frac{d}{d t} \chi_{j}(t, x)\right] d x \\
& +\int_{\mathbb{R}^{s}} \sum_{j=1}^{n} \frac{\partial F_{\varphi_{0}}}{\partial z_{j}}(x, \chi(t, x)) \frac{d}{d t} \chi_{j}(t, x) d x \equiv I_{1}+I_{2}+I_{3} .
\end{aligned}
$$

Application of the mean value theorem yields

$$
\begin{aligned}
I_{1}= & \sum_{j=1}^{n} \int_{\mathbb{R}^{s}}\left\{\int _ { 0 } ^ { 1 } \left[\frac{\partial F_{\varphi_{0}}}{\partial z_{j}}(x, \chi(t, x)+\sigma(\chi(t+\Delta t, x)-\chi(t, x))]\right.\right. \\
& \left.-\frac{\partial F_{\varphi_{0}}}{\partial z_{j}}(x, \chi(t, x))\right\} d \sigma \cdot \frac{\chi_{j}(t+\Delta t, x)-\chi_{j}(t, x)}{\Delta t} d x
\end{aligned}
$$

and therefore $I_{1} \rightarrow 0$, as $\Delta t \rightarrow 0$, since $F_{\varphi_{0}} \in \mathscr{U}(Y)$,

$$
\sup _{|\Delta t| \leqq 1}\left\|\frac{\chi(t+\Delta t, x)-\chi(t, x)}{\Delta t} ; Y_{0}\right\|<\infty
$$


and $\left\|\chi(t+\Delta t, x)-\chi(t, x) ; Y_{1}\right\|_{\Delta t \rightarrow 0} \rightarrow 0$. A similar argument takes care of the term $I_{2}$. In conclusion

$$
d C(t) / d t=<d \chi(t) / d t, \nabla_{z} F_{\varphi}(x, \chi(t))
$$

which implies Equation (B.5) as a consequence of the explicit expression for $d \chi / d t$.

\section{Appendix C}

Throughout the whole Appendix we will work in $\mathbb{R}^{1}$ and suppose $U \in \mathscr{U}(X)$ independent of the $x$ variable. It is convenient to separate the case $n=1$ from the case $n>1$.

Theorem C.1 $(n=1)$. If

i) $\forall \lambda \in \mathbb{R}$ the equation $U(z)-\lambda=0$ has at most a discrete set of solutions

ii) $\forall \lambda \in \mathbb{R}$ one has $\int_{z_{0}}^{\infty}|U(z)-\lambda|^{1 / 2} d z=\int_{-\infty}^{z_{0}}|U(z)-\lambda|^{1 / 2} d z=+\infty$

for some $z_{0} \in \mathbb{R}$ then any $\varphi$ with $\varphi^{\prime} \in L^{2}\left(\mathbb{R}^{1}\right)$ and such that

$$
U(\varphi(x))-\lambda(\varphi) \in L^{1}\left(\mathbb{R}^{1}\right)
$$

for some real constant $\lambda(\varphi)$, has a finite limit as $x \rightarrow \pm \infty$.

Proof. Let us define

$$
A(z)=\int_{z_{0}}^{z}|U(\sigma)-\lambda(\varphi)|^{1 / 2} d \sigma .
$$

Then, the fundamental theorem of calculus and the Schwarz inequality yield

$$
|A(\varphi(x))-A(\varphi(y))| \leqq\left|\int_{y}^{x}\right| U(\varphi(\sigma))-\lambda(\varphi)|d \sigma|^{1 / 2}\left|\int_{y}^{x}\left(\varphi^{\prime}(\sigma)\right)^{2} d \sigma\right|^{1 / 2}
$$

which implies that $A(\varphi(x))$ has finite limits $a_{+}, a_{-}$as $x \rightarrow+\infty,-\infty$. The function $A(z)$ is strictly increasing since $A^{\prime}(z)=|U(z)-\lambda(\varphi)|^{1 / 2}$ vanishes only on a discrete set by condition i). Moreover, by condition ii), range of $A=\mathbb{R}$.

Consequently

$$
\varphi(x)=A^{-1}\left(A(\varphi(x)) \underset{x \rightarrow \pm \infty}{\longrightarrow} A^{-1}\left(a_{ \pm}\right) .\right.
$$

Theorem C.2 $(n>1)$. If

i) $\forall \lambda \in \mathbb{R}$ the equation $\min _{|z|=\varrho}\left|U\left(z_{1}, \ldots, z_{n}\right)-\lambda\right|=0$ has at most a discrete set of solutions $\varrho$.

ii) $\forall \lambda \in \mathbb{R} \int_{0}^{\infty}\left(\min _{|z|=\varrho}\left|U\left(z_{1}, \ldots, z_{n}\right)-\lambda\right|\right)^{1 / 2} d \varrho=+\infty$.

Then, for any $\varphi=\left(\varphi_{1}, \ldots, \varphi_{n}\right)$ with $\varphi_{j}^{\prime} \in L^{2}\left(\mathbb{R}^{1}\right), \forall j$ and such that

$$
U\left(\varphi_{1}(x), \ldots, \varphi_{n}(x)\right)-\lambda(\varphi) \in L^{1}\left(\mathbb{R}^{1}\right)
$$

for some real constant $\lambda(\varphi),|\varphi(x)| \equiv\left(\sum_{j=1}^{n} \varphi_{j}(x)^{2}\right)^{1 / 2}$ has a finite limit as $x \rightarrow \pm \infty$. 
Proof. With the definition

$$
A(\tau)=\int_{0}^{\tau} F(\varrho) d \varrho
$$

where

$$
F(\varrho)=\left(\min _{|z|=\varrho}\left|U\left(z_{1}, \ldots, z_{n}\right)-\lambda(\varphi)\right|\right)^{1 / 2}
$$

the argument proceeds as in Theorem C.1.

The conditions on $\varphi$ in Theorems C.1 and C.2 are satisfied if $\varphi_{j}^{\prime} \in L^{2}\left(\mathbb{R}^{1}\right) \forall j$ and

$$
\varphi_{j}^{\prime \prime}(x)=\frac{\partial U}{\partial z_{j}}\left(\varphi_{1}(x), \ldots, \varphi_{n}(x)\right), j=1, \ldots, n .
$$

In fact Equation (C.4) implies the existence of a constant $\lambda(\varphi)$ such that

$$
U\left(\varphi_{1}, \ldots, \varphi_{n}\right)-\lambda(\varphi)=\frac{1}{2} \sum_{j=1}^{n}\left(\varphi_{j}^{\prime}(x)\right)^{2} \in L^{1}\left(\mathbb{R}^{1}\right) .
$$

Now, if $U\left(\varphi_{1}, \ldots, \varphi_{n}\right)$ has a finite limit as $x \rightarrow \pm \infty$, then necessarily $\lim _{x \rightarrow \pm \infty} U\left(\varphi_{1}(x), \ldots, \varphi_{n}(x)\right)=\lambda(\varphi)$. This obviously happens if the $\varphi_{j}^{\prime} s$ have a finite limit as $x \rightarrow \pm \infty$ or if $|\varphi(x)|$ has a finite limit in the case $U(z)=U(|z|)$. The following example shows that additional assumptions on $U$, such as conditions i) and ii) are necessary in order to guarantee that $\varphi$ has a finite limit. If we have $U(z)=2 e^{-z}\left(1-e^{-z}\right)$ for $z \geqq 0, U=2 z-3 z^{2}$ for $z \leqq 0, \varphi(x)=\ln \left(1+x^{2}\right)$, then $\varphi^{\prime \prime}(x)=$ $\left(\frac{d U}{d z}\right)(\varphi(x)), \varphi^{\prime} \in L^{2}\left(\mathbb{R}^{1}\right)$ but $\varphi(x) \rightarrow+\infty$ as $x \rightarrow \pm \infty$.

For $n=1$ examples of potentials $U(z)$ satisfying conditions i) and ii) of Theorem C. 1 are the polynomials of degree $\geqq 1, U(z)=\cos z, U(z)=e^{z^{2}}$. For the cases $U(z)=\left(z^{2}-1\right)^{2}, U(z)=\cos z$, simple solutions $\varphi$ of Equation (C.4) are the standard soliton-solutions (see $[1,3]$ ).

\section{References}

1. Lee, T. D., Wick, G. C.: Phys. Rev. D9, 2291 (1974)

Ferretti, B.: Atti Acc. Scienze Bologna, Serie XII, Tomo I, 45 (1974)

't Hooft, G.: Nucl. Phys. B79, 276 (1974)

Ferretti, B., Velo, G. : Nuovo Cimento Letters 10, 451 (1974)

Dashen, R., Hasslacher, B., Neveu, A.: Phys. Rev. D10, 4114, 4130, 4138 (1974)

Goldstone, J., Jackiw, R.: Phys. Rev. D11, 1486 (1975)

Coleman, S.: Phys. Rev. D11, 2088 (1975)

Fröhlich, J.: Phys. Rev. Letters 34, 833 (1975)

Gervais, J. L., Sakita, R.: Phys. Rev. D11, 2943 (1975)

Mandelstam, S.: Phys. Rev. D11, 3026 (1975)

Fadeev, L. D.: IAS lectures and references therein, April 1975

Christ, N., Lee, T. D.: Phys. Rev. D12, 1606 (1975)

2. Goldstone, J.: Nuovo Cimento 19, 154 (1961)

3. Whitham, G. B.: Linear and nonlinear waves. New York: Wiley 1974

4. Finkelstein, D., Misner, C. W.: Ann. Phys. 6, 230 (1959)

Finkelstein, D.: J. Math. Phys. 7, 1218 (1966) 
5. Hepp, K.: Commun. math. Phys. 35, 265 (1974)

6. Parenti, C., Strocchi, F., Velo, G.: preprint S.N.S. 22/1975, to appear in Ann. Scuola Norm. Sup. (Pisa). A brief account has been given in Phys. Lett. 59B, 157 (1975)

7. Segal, I.: Ann. Math. 78, 339 (1963)

8. Volevic, L. R., Paneyakh, B. P.: Russian Math. Surveys 20, 1 (1965)

9. Gel'fand,I.M., Shilov, G.E.: Generalized Functions, Vol. 2. New York, London: Academic Press 1968

10. Halmos, P. R.: Measure Theory. New York: Van Nostrand 1950

11. Yoshida, K.: Functional Analysis. Berlin-Heidelberg-New York: Springer 1966

Communicated by J. Glimm

Received April 10, 1976 